\title{
EQUIDISTRIBUTION OF RATIONAL MATRICES IN THEIR CONJUGACY CLASSES
}

\author{
YVES BENOIST AND HEE OH
}

\begin{abstract}
Let $\mathbf{G}$ be a connected simply connected almost $\mathbb{Q}$-simple algebraic group with $G:=\mathbf{G}(\mathbb{R})$ non-compact and $\Gamma \subset \mathbf{G}_{\mathbb{Q}}$ a cocompact congruence subgroup. For any homogeneous manifold $x_{0} H \subset \Gamma \backslash G$ of finite volume, and $a \in \mathbf{G}_{\mathbb{Q}}$, we show that the Hecke orbit $\mathrm{T}_{a}\left(x_{0} H\right)$ is equidistributed on $\Gamma \backslash G$ as $\operatorname{deg}(a) \rightarrow \infty$, provided $H$ is a non-compact commutative reductive subgroup of $G$. As a corollary, we generalize the equidistribution result of Hecke points ([COU], [EO1]) to homogeneous spaces $G / H$. As a concrete application, we describe the equidistribution result in the rational matrices with a given characteristic polynomial.
\end{abstract}

\section{INTRODUCTION}

1.1. General setting. Let $\mathbf{G}$ be a connected almost $\mathbb{Q}$-simple algebraic $\mathbb{Q}$-group, $G$ the identity component of $\mathbf{G}_{\mathbb{R}}$ and $\Gamma$ a congruence subgroup in $G_{\mathbb{Q}}:=G \cap \mathbf{G}_{\mathbb{Q}}$. We suppose that $G$ is non-compact. According to a classical result of Borel and HarishChandra in $[\mathrm{BH}], \Gamma$ is a lattice in $G$, i.e., $\Gamma$ has a finite co-volume in $G$. Denote by $\mu_{X}$ the $G$-invariant probability measure on $X:=\Gamma \backslash G$ and by $\pi: G \rightarrow X$ the standard projection.

We recall the Hecke correspondence. For any $a \in G_{\mathbb{Q}}$, the set $\Gamma a \Gamma$ is a finite union of distinct $\Gamma$-orbits:

$$
\Gamma a \Gamma=\bigcup_{1 \leq i \leq \operatorname{deg}(a)} \Gamma a_{i}
$$

where $\operatorname{deg}(a)$ is the degree of $a$. For $x=\pi(g) \in X$, the finite set

$$
\mathrm{T}_{a}(x):=\pi\left(a \pi^{-1}(x)\right)=\left\{\pi\left(a_{i} g\right) \mid i=1, \cdots, \operatorname{deg}(a)\right\}
$$

is called the Hecke orbit of $x$.

According to Clozel-Oh-Ullmo's equidistribution theorem, these sets $\mathrm{T}_{a}(x)$ become equidistributed in $X$ with respect to $\mu_{X}$ as $\operatorname{deg}(a) \rightarrow \infty$ ([COU] and [EO1]).

Let $H \subset G$ be a closed subgroup and $\lambda_{H}$ a left Haar measure on $H$. For any $H$-orbit $Y:=x_{0} H \subset X$ with finite volume, we define the Hecke orbit $\mathrm{T}_{a}(Y)$ of $Y$ :

$$
\mathrm{T}_{a}(Y):=\pi\left(a \pi^{-1}(Y)\right) .
$$

The second author partially supported by DMS 0333397 . 
Noting that $Y$ is closed (Theorem 1.13 of $[\mathrm{Ra}]$ ), $\mathrm{T}_{a}(Y)$ is a finite union of closed $H$ orbits of finite volume. Let $\lambda_{a}$ denote the unique $H$-invariant measure on $X$ supported on $\mathrm{T}_{a}(Y)$ which is locally equal to $\lambda_{H}$ on each $H$-orbit in $\mathrm{T}_{a}(Y)$.

Theorem 1.1. (Equidistribution for $\mathrm{T}_{a}(Y)$ ) Let $\mathbf{G}$ be a connected simply connected almost $\mathbb{Q}$-simple algebraic $\mathbb{Q}$-group with $G=\mathbf{G}_{\mathbb{R}}$ non-compact and $\Gamma$ a cocompact congruence subgroup in $\mathbf{G}_{\mathbb{Q}}$. Let $H$ be a closed commutative reductive non-compact subgroup of $G$.

Then for any $H$-orbit $x_{0} H \subset X$ with finite volume, the Hecke orbit $\mathrm{T}_{a}\left(x_{0} H\right)$ becomes equidistributed with respect to $\mu_{X}$ as $\operatorname{deg}(a) \rightarrow \infty$.

That is, for any $f \in \mathrm{C}_{c}(X)$, one has

$$
\lim _{\operatorname{deg}(a) \rightarrow \infty} \frac{1}{\lambda_{a}(X)} \int_{X} f d \lambda_{a}=\int_{X} f d \mu_{X} .
$$

We remark that $\lambda_{a}(X) \leq \lambda_{H}(Y) \cdot \operatorname{deg}(a)$ and that the condition $\operatorname{deg}(a) \rightarrow \infty$ is equivalent to $\lambda_{a}(X) \rightarrow \infty$ (see Corollary 7.3). Note that $\lambda_{a}(X)$ is simply the total volume of $\mathrm{T}_{a}(Y)$.

Recall that a commutative subgroup $H$ is said reductive if all the elements in $H$ are semisimple. There are many examples of $x_{0} H$ which satisfy the assumptions of Theorem 1.1. For instance, for $x_{0}=[e], H$ can be the group of real points of a maximal $\mathbb{R}$-isotropic torus of $\mathbf{G}$ which is $\mathbb{Q}$-anisotropic. Such a Cartan subgroup $H$ always exists [PR1].

The hypothesis " $\Gamma$ cocompact" and the hypothesis " $H$ commutative reductive and non-compact" might be avoided in Theorem 1.1: for instance, when $H$ is maximal semisimple instead of commutative, a similar statement can be obtained as a special case of a general equidistribution statement for $H$-invariant subsets in $\Gamma \backslash G$ due to Eskin and $\mathrm{Oh}$ in [EO2]. Their proof is based on Ratner's classification of $H$-invariant measure on $\Gamma \backslash G$

In addition to Clozel-Oh-Ullmo's theorem [COU], our proof of Theorem 1.1 is based on the introduction of weighted Hecke operators as well as on a careful analysis of the corresponding weights, which allows us to relate them to the standard Hecke operators.

Note that other kinds of related equidistribution statements are due to Linnik [Li], Sarnak [Sa] and Gan and Oh [GO].

The following corollary is a restatement of Theorem 1.1, in view of the homeomorphism between the $H$-invariant measures on $\Gamma \backslash G$ and the $\Gamma$-invariant measures on $G / H$.

Let $V=G / H$ and $v_{0}=g_{0} H \in V$ with $\pi\left(g_{0}\right)=x_{0}$. The $H$-orbit $x_{0} H$ being closed in $X$ implies that $v_{0} \Gamma$ is discrete in $V$. Starting from the single $\Gamma$-orbit $\Gamma v_{0}$ in $V$, the Hecke correspondence allows us to construct the set

$$
\mathrm{T}_{a}\left(v_{0}\right):=\Gamma a \Gamma v_{0} \subset V
$$


which is a finite union of discrete $\Gamma$-orbits for any $a \in G_{\mathbb{Q}}$. Denote by $\lambda$ a $G$-invariant measure on $V$, which is unique up to a scalar multiple.

The following corollary generalizes the equidistribution of Hecke points on $G$ [COU] to homogeneous spaces $G / H$ :

Corollary 1.2. (Equidistribution for $\mathrm{T}_{a}\left(v_{0}\right)$ ) Let $G, H x_{0}=\pi\left(g_{0}\right)$ and $\Gamma$ be as in Theorem 1.1. Then for any compact subset $\Omega$ of $V$ with boundary measure zero, one has for $v_{0}=g_{0} H \in V$,

$$
\#\left(\mathrm{~T}_{a}\left(v_{0}\right) \cap \Omega\right) \sim c_{a} \lambda(\Omega) \quad \text { as } \operatorname{deg}(a) \rightarrow \infty
$$

where $c_{a}=c_{a}(\Gamma)$ are constants independent of $\Omega$.

The most natural family of examples of homogeneous spaces $G / H$ to which Corollary 1.2 applies are the conjugacy classes of regular semisimple elements in $G_{\mathbb{Q}}$. For $s \in G$, denote by $\mathcal{C}(s)$ the conjugacy class of $s$, that is,

$$
\mathcal{C}(s):=\left\{t \in G \mid t=g s g^{-1} \text { for some } g \in G\right\} .
$$

The following says that the set of elements in $C(s)$ which can be conjugate to $s$ via $\Gamma a \Gamma$ is equidistributed:

Corollary 1.3. (Equidistribution in conjugacy class) Let $G$ and $\Gamma$ be as in Theorem 1.1. Let $s \in G_{\mathbb{Q}}$ be a regular semisimple element whose centralizer in $\mathbf{G}$ is a $\mathbb{R}$-isotropic and $\mathbb{Q}$-anisotropic torus.

Then for any compact subset $\Omega$ of $\mathcal{C}(s)$ with boundary measure zero,

$$
\#\left\{t \in \Omega \mid t=\mathrm{gsg}^{-1} \text { for some } g \in \Gamma a \Gamma\right\} \sim c_{a} \lambda(\Omega) \quad \text { as } \operatorname{deg}(a) \rightarrow \infty
$$

where $c_{a}=c_{a}(\Gamma)$ are constants and $\lambda$ denote a $G$-invariant measure on $\mathcal{C}(s)$.

Remark One can remove the hypothesis $\mathbf{G}$ simply connected and $\Gamma$ cocompact in Theorem 1.1 and Corollaries $1.2,1.3$ by considering $G=\mathbf{G}(\mathbb{R})^{\circ}$ and only sequences of elements $a \in G_{\mathbb{Q}}$ with $\# \operatorname{supp}(a)$ uniformly bounded, where $\operatorname{supp}(a)=\left\{p \mid a \notin \mathbf{G}_{\mathbb{Z}_{p}}\right\}$ roughly means the set of primes which appear in the denominators of the coefficients of $a$ (see Theorem 5.5 and Remark 8.3).

1.2. Example. We now give a concrete application of Corollary 1.2 and the above remark.

Consider an irreducible polynomial $P(T)=T^{N}+a_{N-1} T^{N-1}+\cdots+a_{0}$ with integral coefficients with $N \geq 3$. Let $\alpha \in \mathbb{C}$ be a root of $P$. Let

$$
V:=\left\{M \in \mathcal{M}_{N}(\mathbb{R}) \mid P(M)=0\right\}
$$

be the variety of real matrices with characteristic polynomial $P$. The set $V_{\mathbb{Q}}:=$ $V \cap \mathcal{M}_{N}(\mathbb{Q})$ is dense in $V$. We will now define natural discrete subsets $V_{m}$ of $V_{\mathbb{Q}}$ indexed by $N$-tuples $m=\left(m_{1}, \cdots, m_{N}\right)$ of positive integers which we will show become equidistributed on $V$. These subsets $V_{m}$ are defined in terms of the depth of the eigenvector for $\alpha$ in the ring $\mathbb{Z}[\alpha]$. 
More precisely, for $M \in V_{\mathbb{Q}}$, let $\xi_{M}=\left(\xi_{1}, \ldots, \xi_{N}\right) \in \mathbb{Z}[\alpha]^{N}$ be an eigenvector for the eigenvalue $\alpha$ i.e., $M \xi_{M}=\alpha \xi_{M}$ and let $\Lambda_{\xi_{M}}$ be the finite index $\mathbb{Z}$-submodule of $\mathbb{Z}[\alpha]$ given by

$$
\Lambda_{\xi_{M}}:=\oplus_{i=1}^{N} \mathbb{Z} \xi_{i}
$$

Since $P$ is irreducible, $\xi_{M}$ as well as $\Lambda_{\xi_{M}}$ are uniquely determined up to multiplication by $\mathbb{Q}(\alpha)^{*}$. Considering the equivalence relation in the space of $\mathbb{Z}$-submodules of $\mathbb{Z}[\alpha]$ given by the homothety by $\mathbb{Q}(\alpha)^{*}$, we have associated to each $M \in V_{\mathbb{Q}}$ a finite index $\mathbb{Z}$-submodule class $\left[\Lambda_{\xi_{M}}\right]$ of $\mathbb{Z}[\alpha]$ (cf. [LM], [T] and $[\mathrm{S}]$ ). For $m=\left(m_{1}, \ldots, m_{N}\right)$ with $m_{i} \in \mathbb{N}, m_{i+1} \mid m_{i}$ for each $i$ and $m_{N}=1$, we define

$$
V_{m}:=\left\{M \in V_{\mathbb{Q}} \mid \Lambda_{\xi_{M}} \sim \Lambda, \mathbb{Z}[\alpha] / \Lambda \simeq \oplus_{i=1}^{N} \mathbb{Z} / m_{i} \mathbb{Z}\right\} .
$$

For instance, if $p$ is prime, we have

$$
V_{(p, 1, \cdots, 1)}=\left\{M \in V_{\mathbb{Q}} \mid \Lambda_{\xi_{M}} \sim \Lambda,[\mathbb{Z}[\alpha]: \Lambda]=p .\right\}
$$

Then $V_{m}$ is a discrete subset of $V$ and $V_{\mathbb{Q}}$ is the union of the $V_{m}$ 's. The following statement says that $V_{m}$ becomes equidistributed on $V$ with respect to a $\mathrm{GL}_{N}(\mathbb{R})$ invariant measure $\lambda$ :

Proposition 1.4. (Equidistribution for $V_{m}$ ) For any compact subset $\Omega$ of $V$ with boundary measure zero, one has, as $m$ going to infinity with the number of prime divisors of $m$ uniformly bounded,

$$
\#\left(V_{m} \cap \Omega\right) \sim c_{m} \lambda(\Omega)
$$

where $c_{m}$ are constants depending only on $m$.

The hypothesis on the integers $m$ in the above proposition is satisfied when $m$ describes the sequence of prime numbers or the sequence of powers of a fixed prime. We believe that this hypothesis is unnecessary, but the methods in this paper do not apply. Another very interesting open question is whether the sequence $V\left(\frac{\mathbb{Z}}{m}\right)$ is equidistributed on $V$ as $m \rightarrow \infty$.

On the organization of the paper: Given $x_{0} \in X$ and a closed subgroup $H$ of $G$ such that $x_{0} H$ has a finite volume, we introduce in section 2 the multiplicities of Hecke points and the weighted Hecke operators $\widetilde{\mathrm{T}}_{a}=\widetilde{\mathrm{T}}_{a}^{H}$. In section 3 , we give an analogous definition for local $p$ multiplicities for each prime $p$ and relate them with the (global) multiplicities of Hecke points. In section 4, assuming that $H$ is commutative and reductive, we prove that the multiplicities of Hecke points in $\mathrm{T}_{a}\left(x_{0}\right)$ are uniformly bounded if the cardinalities of the support of $a$ are uniformly bounded (Proposition 4.5). In section 5, from Proposition 4.5 we deduce Theorem 1.1 for the case when the cardinalities of $\operatorname{supp}(a)$ are uniformly bounded and $H$ non-compact, based on ergodic theoretic argument. For this part, we do not use the assumption $\mathbf{G}$ simply connected or $\Gamma$ cocompact.

When the cardinality of $\operatorname{supp}(a)$ goes to infinity, we will approximate the weighted Hecke operator $\widetilde{\mathrm{T}}_{a}$ by the product $\widetilde{\mathrm{T}}_{b} \mathrm{~T}_{c}$ of a weighted Hecke operator and a Hecke 
operator (Theorem 7.1). In doing so, a crucial step is to show that for $H$ commutative and reductive, most Hecke points in $\mathrm{T}_{a}\left(x_{0}\right)$ has local $p$-multiplicity 1 for almost all $p$, which is the main theorem of section 6 (Theorem 6.1). Assuming now $\Gamma$ cocompact, we then complete a proof of Theorem 1.1, using Theorem 7.1 and equidistribution results for the standard Hecke operators, in section 7. In section 8, we prove Corollaries 1.2 and 1.3 using the duality between $H$-invariant measures and $\Gamma$-invariant measures.

Acknowledgment We would like to thank Elon Lindenstrauss for useful discussions. The first named author would like to thank Caltech where most of the collaboration took place.

\section{Weighted Hecke operator}

As in section 1.1 , let $\mathbf{G}$ be a connected almost $\mathbb{Q}$-simple algebraic $\mathbb{Q}$-group, $G$ the identity component of $\mathbf{G}_{\mathbb{R}}, G_{\mathbb{Q}}=G \cap \mathbf{G}_{\mathbb{Q}}$ and $\Gamma \subset G_{\mathbb{Q}}$ a congruence subgroup. We assume that $G$ is non-compact. Set $X=\Gamma \backslash G$ and let $\pi: G \rightarrow X$ denote the canonical projection.

Let $H$ be a closed subgroup of $G$, and consider an $H$-orbit $Y:=x_{0} H \subset X$ with finite volume for $x_{0}=\pi\left(g_{0}\right) \in X$. For the sake of simplicity, we will suppose that $g_{0}=e$ and hence that $H_{x_{0}}:=H \cap \Gamma$ is the stabilizer of $x_{0}$ in $H$ (the general case can be reduced to this case simply by replacing $H$ with $\left.g_{0} H g_{0}^{-1}\right)$.

2.1. Standard Hecke operators. For $a \in G_{\mathbb{Q}}$, the Hecke orbit of $x_{0}$ is

$$
\mathrm{T}_{a}\left(x_{0}\right)=\pi(a \Gamma)=\left\{x_{i} \mid i=1, \ldots, \operatorname{deg}(a)\right\}
$$

where $x_{i}:=\pi\left(a_{i}\right)$ with $a_{i} \in G_{\mathbb{Q}}$. It is easy to see that $\operatorname{deg}(a)=\left[\Gamma: a^{-1} \Gamma a \cap \Gamma\right]$.

The standard Hecke operator $\mathrm{T}_{a}: \mathrm{C}_{c}(X) \rightarrow \mathrm{C}_{c}(X)$ is defined as follows: for any $f \in \mathrm{C}_{c}(X)$ and $g \in G$,

$$
\mathrm{T}_{a} f(\pi(g))=\frac{1}{\operatorname{deg}(a)} \sum_{1 \leq i \leq \operatorname{deg}(a)} f\left(x_{i} g\right) .
$$

Recall the equidistribution theorem for the Hecke points:

Theorem 2.1. ([COU], [EO1]) For any $f \in \mathrm{C}_{c}(X)$ and any $x \in X$,

$$
\lim _{\operatorname{deg}(a) \rightarrow \infty} \mathrm{T}_{a} f(x)=\int_{X} f d \mu_{X},
$$

this limit being uniform for $x$ in the compact subsets of $X$.

In other words, the uniformly distributed probability measures

$$
\delta_{a}(x):=\frac{1}{\operatorname{deg}(a)} \sum_{x^{\prime} \in \mathrm{T}_{a}(x)} \delta_{x^{\prime}}
$$


on the finite sets $\mathrm{T}_{a}(x)$ converge to $\mu_{X}$ when $\operatorname{deg}(a) \rightarrow \infty$.

2.2. Weighted Hecke operators. Let $a \in G_{\mathbb{Q}}$. For each $x \in \mathrm{T}_{a}\left(x_{0}\right)$, we define its multiplicity with respect to $H$ by

$$
m(x, H):=\#(\Sigma(x, H)) \geq 1
$$

where

$$
\Sigma(x, H):=\mathrm{T}_{a^{-1}}(x) \cap x_{0} H=\left\{x_{0}^{\prime} \in x_{0} H \mid x \in \mathrm{T}_{a}\left(x_{0}^{\prime}\right)\right\} .
$$

It is easy to check that for $x=\pi(g)$,

$$
\Sigma(x, H)=\pi\left(H \cap \mathbf{G}_{\mathbb{Q}}\right) \cap \pi\left(g^{-1} \Gamma g\right) .
$$

We will therefore write $\Sigma\left(x, H \cap \mathbf{G}_{\mathbb{Q}}\right)$ and $m\left(x, H \cap \mathbf{G}_{\mathbb{Q}}\right)$, or even $\Sigma(x)$ and $m(x)$ for simplicity.

Indeed, these multiplicities $m(x)$ depend only on the $H$-orbit $\omega$ of $x$, which will be clear from more intuitive and geometric formulas for $m(x)=m_{\omega}$ (see (2.5) and (2.7)).

Definition 2.2. We define the weighted Hecke operator $\widetilde{\mathrm{T}}_{a}^{H}: \mathrm{C}_{c}(X) \rightarrow \mathrm{C}_{b}(G)$ with respect to $H$ as follows: for any $f \in \mathrm{C}_{c}(X)$ and $g \in G$,

$$
\widetilde{\mathrm{T}}_{a}^{H} f(g)=\frac{1}{\widetilde{\operatorname{deg}}^{H}(a)} \sum_{1 \leq i \leq \operatorname{deg}(a)} \frac{1}{m\left(x_{i}\right)} f\left(x_{i} g\right)
$$

where $\widetilde{\operatorname{deg}}^{H}(a)$ denotes the weighted degree:

$$
\widetilde{\operatorname{deg}}^{H}(a):=\sum_{1 \leq i \leq \operatorname{deg}(a)} \frac{1}{m\left(x_{i}\right)} .
$$

For simplicity, we will write $\widetilde{\mathrm{T}}_{a}$ and $\widetilde{\operatorname{deg}}(a)$, omitting the superscript $H$ whenever it is clear. Note that $\widetilde{\mathrm{T}}_{a} f$ is a bounded continuous function on $G$ for $f \in C_{c}(G)$ and $\widetilde{\mathrm{T}}_{a} f$ is identity on constant functions.

2.3. Weights and Hecke image of $H$-orbit. The main motivation for introducing the weighted Hecke operators $\widetilde{\mathrm{T}}_{a}$ is the following lemma.

As in the introduction, $\lambda_{a}=\lambda_{a}^{H}$ denotes the unique measure on $X$ supported on $\mathrm{T}_{a}(Y)$ locally equal to $\lambda_{H}$ on each $H$-orbit in $\mathrm{T}_{a}(Y)$ and $\mu_{a}=\mu_{a}^{H}$ denotes the probability measure which is proportional to $\lambda_{a}$. In the same way, denote by $\lambda_{Y}$ be the unique $H$-invariant measure on $X$ supported on $Y$ locally equal to $\lambda_{H}$ and by $\mu_{Y}$ the probability measure on $X$ proportional to $\lambda_{Y}$.

Lemma 2.3. Let $f \in \mathrm{C}_{c}(X)$ and $a \in G_{\mathbb{Q}}$.

a) For any $g \in G$ and $h \in H_{x_{0}}$, one has

$$
\widetilde{\mathrm{T}}_{a} f(h g)=\widetilde{\mathrm{T}}_{a} f(g) .
$$


Hence, $\widetilde{\mathrm{T}}_{a}$ can be considered as an operator $\widetilde{\mathrm{T}}_{a}: C_{c}(X) \rightarrow C_{b}\left(H_{x_{0}} \backslash G\right)$.

b) One has $\lambda_{a}(X)=\widetilde{\operatorname{deg}}(a) \lambda_{Y}(X)$.

c) One has $\mu_{a}(f)=\int_{Y} \widetilde{\mathrm{T}}_{a} f(y) d \mu_{Y}(y)$.

Remark The claim b) says that the weighted degree is nothing but the ratio of the volumes of $\mathrm{T}_{a}(Y)$ and $Y$.

Proof Recall that $x_{0}=\pi(e)$.

a) Note that, for $h \in H_{x_{0}}$ and $x_{i} \in \mathrm{T}_{a}\left(x_{0}\right)$, we have $x_{i} h \in \mathrm{T}_{a}\left(x_{0}\right)$ and hence $x_{i} h=x_{j}$ for some $1 \leq j \leq \operatorname{deg}(a)$. It follows from the definitions that for any $h \in H_{x_{0}}$,

$$
\Sigma\left(x_{j}\right)=\Sigma\left(x_{i}\right) h \quad \text { and } \quad m\left(x_{j}\right)=m\left(x_{i}\right) .
$$

One can then compute

$$
\begin{aligned}
\widetilde{\mathrm{T}}_{a} f(h g) & =\widetilde{(\operatorname{deg}}(a))^{-1} \sum_{1 \leq i \leq \operatorname{deg}(a)} \frac{1}{m\left(x_{i}\right)} f\left(x_{i} h g\right) \\
& =\widetilde{(\operatorname{deg}(a))^{-1}} \sum_{1 \leq j \leq \operatorname{deg}(a)} \frac{1}{m\left(x_{j}\right)} f\left(x_{j} g\right) \\
& =\widetilde{\mathrm{T}}_{a} f(g) .
\end{aligned}
$$

b) Let

$$
X_{a}:=\{(x, y) \in X \times X \mid \text { there exists } g \in G \text { with } x=\pi(g) \text { and } y=\pi(a g)\} .
$$

As a homogeneous space, one has $X_{a} \simeq G /\left(\Gamma \cap a^{-1} \Gamma a\right)$. Consider the projections $\pi_{1}, \pi_{2}: X_{a} \rightarrow X$ given by

$$
\pi_{1}(x, y)=x \quad \text { and } \quad \pi_{2}(x, y)=y
$$

which are coverings of degree $\operatorname{deg}(a)$. Then

$$
\mathrm{T}_{a}(x)=\pi_{2}\left(\pi_{1}^{-1}(x)\right) \quad \text { and } \quad \mathrm{T}_{a}(Y)=\pi_{2}\left(\pi_{1}^{-1}(Y)\right)
$$

For the finite union $Z:=\pi_{1}^{-1}(Y)$ of $H$-orbits, denote by $\lambda_{Z}$ the $H$-invariant measure on $Z$ which is given locally by $\lambda_{H}$. Fixing an $H$-orbit $\omega=x_{i} H$ in $\mathrm{T}_{a}(Y)$, let

$$
Z_{\omega}:=Z \cap \pi_{2}^{-1}(\omega)
$$

Then $Z_{\omega}$ is also a finite union of $H$-orbits.

Consider the following diagram:

$$
\operatorname{degree}\left(\pi_{1} \mid Z_{\omega}\right)=d_{\omega} \bigvee_{Y \subset X}^{Z_{\omega} \subset X_{a}} \underset{\omega \subset X}{\operatorname{degree}\left(\pi_{2} \mid Z_{\omega}\right)=m_{\omega}}
$$


As in the above diagram, denote by $d_{\omega}$ the degree of the covering map $\left.\pi_{1}\right|_{Z_{\omega}}: Z_{\omega} \rightarrow Y$ and by $m_{\omega}$ the degree of the covering map $\left.\pi_{2}\right|_{Z_{\omega}}: Z_{\omega} \rightarrow \omega$. Note that

$$
d_{\omega}=\#\left(\pi_{1}^{-1}\left(x_{0}\right) \cap Z_{\omega}\right) \quad \text { and } \quad m_{\omega}=\#\left(\pi_{1}^{-1}(Y) \cap \pi_{2}^{-1}\left(x_{i}\right)\right) .
$$

Note that $\# \pi_{1}^{-1}(x)=\operatorname{deg}(a)$. Since $\mathrm{T}_{a}(x)=\pi_{2}^{-1}\left(\pi_{1}^{-1}(x)\right)$, the projection $\pi_{2}$ restricted to each fiber of $\pi_{1}$ is injective. Therefore one has

$$
d_{\omega}=\#\left(\mathrm{~T}_{a}\left(x_{0}\right) \cap \omega\right) .
$$

Similarly,

$$
m_{\omega}=\#\left(Y \cap T_{a^{-1}}\left(x_{i}\right)\right)=m\left(x_{i}\right)
$$

where $m\left(x_{i}\right)$ is the multiplicity of $x_{i}$.

Therefore we have

$$
\lambda_{a}(X)=\sum_{\omega} \lambda_{a}(\omega)=\sum_{\omega} \frac{1}{m_{\omega}} \lambda_{Z}\left(Z_{\omega}\right)=\sum_{\omega} \frac{d_{\omega}}{m_{\omega}} \lambda_{Y}(Y)=\widetilde{\operatorname{deg}}(a) \lambda_{Y}(X)
$$

where the sum is taken over all $H$-orbits $\omega$ in $\mathrm{T}_{a}(Y)$.

c) One follows a similar computation to obtain

$$
\begin{aligned}
\lambda_{a}(f) & =\sum_{\omega} \int_{\omega} f(y) d \lambda_{a}(y)=\sum_{\omega} \frac{1}{m_{\omega}} \int_{Z_{\omega}} f\left(\pi_{2}(z)\right) d \lambda_{Z}(z) \\
& =\sum_{\omega} \frac{1}{m_{\omega}} \int_{Y}\left(\sum_{z \in \pi_{1}^{-1}(y) \cap Z_{\omega}} f\left(\pi_{2}(z)\right)\right) d \lambda_{Y}(y)=\widetilde{\operatorname{deg}}(a) \int_{Y} \widetilde{\mathrm{T}}_{a} f(y) d \lambda_{Y}(y)
\end{aligned}
$$

where the sum is taken over all $H$-orbits $\omega$ included in $\mathrm{T}_{a}(Y)$. Since $\lambda_{a}(X)=$ $\widetilde{\operatorname{deg}}(a) \lambda_{Y}(X)$ by $(\mathrm{b})$, this implies (c).

Remark The same computation as above yields the following formula, which gives the interpretation of the multiplicities $m_{\omega}$ in relation with the standard Hecke operator:

$$
\int_{Y} \mathrm{~T}_{a} f(y) d \lambda_{Y}(y)=\sum_{\omega \in \mathrm{T}_{a}(Y)} m_{\omega} \int_{\omega} f d \lambda_{a}
$$

In the following the convergence of measures on $X$ are understood in terms of weak* topology on the space of probability measures as usual.

Lemma 2.6. Suppose that $Y=x_{0} H$ is compact. Suppose that, for any $f \in \mathrm{C}_{c}(X)$,

$$
\lim _{\operatorname{deg}(a) \rightarrow \infty} \widetilde{\mathrm{T}}_{a} f(g)=\int_{X} f d \mu_{X} \quad \text { uniformly for } g \text { on compact sets of } G \text {. }
$$

Then one has $\lim _{\operatorname{deg}(a) \rightarrow \infty} \mu_{a}=\mu_{X}$. 
Proof This is a straightforward application of Lemma 2.3.c.

Let

$$
X_{a}=\{(x, y) \in X \times X \mid \text { there exists } g \in G \text { with } x=\pi(g) \text { and } y=\pi(a g)\} .
$$

and $\pi_{1}, \pi_{2}: X_{a} \rightarrow X$ be given by $\pi_{i}\left(x_{1}, x_{2}\right)=x_{i}$ for $i=1,2$.

The following lemma about the multiplicity was proven in the course of proof of Lemma 2.3.b.:

Lemma 2.7. Let $x \in \mathrm{T}_{a}\left(x_{0}\right)$ and $\omega=x H$. Then

$$
m(x, H)=m_{\omega}
$$

where $m_{\omega}$ is the degree of the covering map $\pi_{1}^{-1}\left(x_{0} H\right) \cap \pi_{2}^{-1}(\omega) \rightarrow \omega$ induced by the restriction of $\pi_{2}$. That is,

$$
m_{\omega}=\# \pi_{1}^{-1}\left(x_{0} H\right) \cap \pi_{2}^{-1}(x)
$$

\section{Multiplicity AND LOCAL MUltiplicity}

3.1. Multiplicity. Let $\mathbf{G}$ be a connected semisimple $\mathbb{Q}$-group, $G$ the identity component of $\mathbf{G}_{\mathbb{R}}$ and $\Gamma \subset G_{\mathbb{Q}}$ be a congruence subgroup. Set

$$
X_{\mathbb{Q}}:=\Gamma \backslash G_{\mathbb{Q}} \quad \text { and } \quad x_{0}:=\pi(e)
$$

where $\pi: G_{\mathbb{Q}} \rightarrow X_{\mathbb{Q}}$ denotes the standard projection.

Definition 3.1. Let $\Delta$ be a subgroup of $G_{\mathbb{Q}}$. For $x=\pi(g) \in X_{\mathbb{Q}}$, define

$$
\Sigma(x, \Delta):=\pi(\Delta) \cap \pi\left(g^{-1} \Gamma g\right) \subset X_{\mathbb{Q}} .
$$

Note that $\Sigma(x, \Delta)$ is a non-empty finite set. We call its cardinality

$$
m(x, \Delta):=\# \Sigma(x, \Delta) \geq 1
$$

the multiplicity of $x$ with respect to $\Delta$.

Remark When $\Delta=H \cap G_{\mathbb{Q}}$, the sets $\Sigma(x, \Delta)$ and the multiplicities $m(x, \Delta)$ coincide with those defined in section 2.2.

Here are some basic properties of the multiplicities $m(x, \Delta)$.

Lemma 3.2. Let $x \in X_{\mathbb{Q}}$ and $\Delta$ a subgroup of $G_{\mathbb{Q}}$.

a) Let $\Gamma^{\prime} \subset G_{\mathbb{Q}}$ be a congruence subgroup containing $\Gamma$ and $\sigma: X_{\mathbb{Q}} \rightarrow \Gamma^{\prime} \backslash G_{\mathbb{Q}}$ the canonical projection. Then one has

$$
m(x, \Delta) \leq\left[\Gamma^{\prime}: \Gamma\right] m(\sigma(x), \Delta) .
$$

b) If $\Delta^{\prime} \subset G_{\mathbb{Q}}$ is a subgroup containing $\Delta$, then

$$
m(x, \Delta) \leq m\left(x, \Delta^{\prime}\right)
$$


c) Let $\mathbf{G}^{\prime}$ be a connected semisimple $\mathbb{Q}$-group containing $\mathbf{G}, \Gamma^{\prime}$ a congruence subgroup of $G_{\mathbb{Q}}^{\prime}$ and $\Delta^{\prime}$ a subgroup of $G_{\mathbb{Q}}^{\prime}$ such that $\Gamma:=\Gamma^{\prime} \cap G$ and $\Delta:=\Delta^{\prime} \cap G$. Denote by $i: X_{\mathbb{Q}} \rightarrow \Gamma^{\prime} \backslash G_{\mathbb{Q}}^{\prime}$ the canonical injection. Then one has

$$
m(x, \Delta) \leq m\left(i(x), \Delta^{\prime}\right) .
$$

Proof a) Use the inclusion $\sigma(\Sigma(x, \Delta)) \subset \Sigma(\sigma(x), \Delta)$.

b) Use the inclusion $\Sigma(x, \Delta) \subset \Sigma\left(x, \Delta^{\prime}\right)$.

c) Use the inclusion $\Sigma(x, \Delta) \subset \Sigma\left(i(x), \Delta^{\prime}\right)$.

3.2. Local multiplicity. We now define the local analogs of these multiplicities. We denote by $P$ the set of all primes. Let $p \in P, \mathbf{G}$ a connected semisimple $\mathbb{Q}_{p}$-group and $U \subset \mathbf{G}_{\mathbb{Q}_{p}}$ a compact open subgroup of $\mathbf{G}_{\mathbb{Q}_{p}}$. Set $X_{\mathbb{Q}_{p}}:=U \backslash \mathbf{G}_{\mathbb{Q}_{p}}$ and denote by $\pi_{p}: \mathbf{G}_{\mathbb{Q}_{p}} \rightarrow X_{\mathbb{Q}_{p}}$ the canonical projection.

Definition 3.3. For $x=\pi_{p}(g) \in X_{\mathbb{Q}_{p}}$ and a subgroup $\Delta$ of $\mathbf{G}_{\mathbb{Q}_{p}}$, define

$$
\begin{gathered}
\Sigma_{p}(x, \Delta):=\pi_{p}(\Delta) \cap \pi_{p}\left(g^{-1} U g\right) \subset X_{\mathbb{Q}_{p}} ; \\
m_{p}(x, \Delta):=\#\left(\Sigma_{p}(x, \Delta)\right)<\infty .
\end{gathered}
$$

We call $m_{p}(x, \Delta)$ the local multiplicity of $x$ at $p$ with respect to $\Delta$.

The following lemma is similar to Lemma 3.2 for the local multiplicities.

Lemma 3.4. Let $x \in X_{\mathbb{Q}_{p}}$ and $\Delta$ a subgroup of $\mathbf{G}_{\mathbb{Q}_{p}}$.

a) Let $U^{\prime} \subset \mathbf{G}_{\mathbb{Q}_{p}}$ be a compact open subgroup containing $U$ and $\sigma_{p}: X_{\mathbb{Q}_{p}} \rightarrow U^{\prime} \backslash \mathbf{G}_{\mathbb{Q}_{p}}$ the canonical projection. Then one has

$$
m_{p}(x, \Delta) \leq\left[U^{\prime}: U\right] m_{p}\left(\sigma_{p}(x), \Delta\right) .
$$

b) If $\Delta^{\prime} \subset \mathbf{G}_{\mathbb{Q}_{p}}$ is a subgroup containing $\Delta$, then

$$
m_{p}(x, \Delta) \leq m_{p}\left(x, \Delta^{\prime}\right) .
$$

c) Let $\mathbf{G}^{\prime}$ be a connected semisimple $\mathbb{Q}_{p}$-group containing $\mathbf{G}, U^{\prime}$ a compact open subgroup of $\mathbf{G}_{\mathbb{Q}_{p}}^{\prime}$ and $\Delta^{\prime}$ a subgroup of $\mathbf{G}_{\mathbb{Q}_{p}}^{\prime}$ such that $U:=U^{\prime} \cap \mathbf{G}$ and $\Delta:=\Delta^{\prime} \cap \mathbf{G}$. Denote by $i: X_{\mathbb{Q}_{p}} \rightarrow U^{\prime} \backslash \mathbf{G}_{\mathbb{Q}_{p}}^{\prime}$ the canonical injection. Then one has

$$
m_{p}(x, \Delta) \leq m_{p}\left(i(x), \Delta^{\prime}\right)
$$

Proof Same proof as Lemma 3.2.

3.3. Structure of $\Gamma$-orbits in $\mathbf{X}_{\mathbb{Q}}$. We now explain the relation between the global and local multiplicities.

Let $\mathbf{G}_{\mathbb{A}_{f}}$ be the group of finite adeles of $\mathbf{G}$. It is the restricted topological product for $p \in P$ of all the groups $\mathbf{G}_{\mathbb{Q}_{p}}$ with respect to the compact open subgroups $\mathbf{G}_{\mathbb{Z}_{p}}$. An element $g$ of $\mathbf{G}_{\mathbb{A}_{f}}$ is then a family $\left(g_{p}\right)_{p \in P}$ of elements of $\mathbf{G}_{\mathbb{Q}_{p}}$ which are in $\mathbf{G}_{\mathbb{Z}_{p}}$ for almost all $p$. For $p \in P$, let $U_{p}$ be a compact open subgroup of $\mathbf{G}_{\mathbb{Q}_{p}}$ which is equal to 
$\mathbf{G}_{\mathbb{Z}_{p}}$ for almost all $p$. The group $U:=\prod_{p \in P} U_{p}$ is a compact open subgroup of $\mathbf{G}_{\mathbb{A}_{f}}$. We will implicitly embed $\mathbf{G}_{\mathbb{Q}}$ diagonally as a subgroup of $\mathbf{G}_{\mathbb{A}_{f}}$.

The homogeneous space $X_{\mathbb{A}_{f}}:=U \backslash \mathbf{G}_{\mathbb{A}_{f}}$ is the restricted product of the local homogeneous spaces $X_{\mathbb{Q}_{p}}:=U_{p} \backslash \mathbf{G}_{\mathbb{Q}_{p}}$, and an element $x$ of $X_{\mathbb{A}_{f}}$ is a family $\left(x_{p}\right)_{p \in P}$ of elements of $X_{\mathbb{Q}_{p}}$ which are equal to the base point $\pi_{p}(e)$ for almost all $p$. We will denote by $\pi_{f}: \mathbf{G}_{\mathbb{A}_{f}} \rightarrow X_{\mathbb{A}_{f}}$ the canonical projection. Recall that for the base point $x_{0}=\pi_{f}(e)$ of $X_{\mathbb{A}_{f}}$ and for $g \in \mathbf{G}_{\mathbb{A}_{f}}$, the Hecke orbit $\mathrm{T}_{g}\left(x_{0}\right) \subset X_{\mathbb{A}_{f}}$ is given by

$$
\mathrm{T}_{g}\left(x_{0}\right)=x_{0} g U
$$

For any $x \in X_{\mathbb{Q}}$, denote by $x_{p}$ its image in $X_{\mathbb{Q}_{p}}$ under the canonical embedding of $X_{\mathbb{Q}}$ into $X_{\mathbb{Q}_{p}}$. Similarly, for any subgroup $\Delta$ of $G_{\mathbb{Q}}$, denote by $\Delta_{p}$ the closure of its image in $\mathbf{G}_{\mathbb{Q}_{p}}$. Let $\widehat{\Delta}$ be the restricted product of the $\Delta_{p}$ 's with respect to the subgroups $\Delta_{p} \cap U_{p}, p \in P$.

Definition 3.5. For $x=\pi(g) \in X_{\mathbb{Q}}$ and a subgroup $\Delta \subset G_{\mathbb{Q}}$, define

$$
\begin{gathered}
\widehat{\Sigma}(x, \Delta):=\pi_{f}(\widehat{\Delta}) \cap \pi_{f}\left(g^{-1} U g\right)=\prod_{p \in P} \Sigma_{p}\left(x_{p}, \Delta_{p}\right) \text { and } \\
\widehat{m}(x, \Delta):=\# \widehat{\Sigma}(x, \Delta)=\prod_{p \in P} m_{p}\left(x_{p}, \Delta_{p}\right)
\end{gathered}
$$

Since $m_{p}\left(x_{p}, \Delta_{p}\right)=1$ whenever $x_{p}=\pi_{p}(e)$, and hence for almost all primes $p \in P$, one has $\widehat{m}(x, \Delta)<\infty$.

Set

$$
\Gamma:=G_{\mathbb{Q}} \cap U
$$

Then $\Gamma$ is a congruence subgroup, and via the injective map $X_{\mathbb{Q}} \rightarrow X_{\mathbb{A}_{f}}$ induced by the diagonal imbedding $G_{\mathbb{Q}} \rightarrow \mathbf{G}_{\mathbb{A}_{\mathbf{f}}}$, we may identify $X_{\mathbb{Q}}$ as a subset of $X_{\mathbb{A}_{f}}$.

The relation between the multiplicities $m(x)$ and $\widehat{m}(x)$ is given by the inequality:

Lemma 3.7. For any $x \in X_{\mathbb{Q}}$ and a subgroup $\Delta \subset G_{\mathbb{Q}}$, one has

$$
m(x, \Delta) \leq \widehat{m}(x, \Delta) .
$$

Proof This results from the inclusion $\Sigma(x, \Delta) \subset \pi_{f}(\Delta) \cap \pi_{f}\left(g^{-1} \Gamma g\right) \subset \widehat{\Sigma}(x, \Delta)$.

3.4. Product of relatively prime elements. In this subsection, we further assume that $\mathbf{G}$ is an almost $\mathbb{Q}$-simple simply connected algebraic $\mathbb{Q}$-group with $\mathbf{G}_{\mathbb{R}}$ noncompact. In particular, it follows that $G=\mathbf{G}_{\mathbb{R}}$, and by the strong approximation theorem that $\mathbf{G}_{\mathbb{Q}}$ is dense in $\mathbf{G}_{\mathbb{A}_{f}}$ (see $[\mathrm{PR}]$ ), and hence $\Gamma$ is dense in $U$.

The following lemma is a basic fact for the adelic interpretation of the Hecke correspondence.

Lemma 3.8 (GO, Lemma 2.3). For any $g \in \mathbf{G}_{\mathbb{Q}}$, one has

$$
\mathbf{G}_{\mathbb{Q}} \cap U g U=\Gamma g \Gamma \quad \text { and } \quad U g \Gamma=U g U .
$$


Since $\Gamma=\mathbf{G}_{\mathbb{Q}} \cap U$, one has

$$
X_{\mathbb{Q}} \simeq U \backslash \mathbf{G}_{\mathbb{A}_{f}}=X_{\mathbb{A}_{f}}
$$

and the $\Gamma$ orbits in $X_{\mathbb{Q}}$ are in bijection with the $U$-orbits in $X_{\mathbb{A}_{f}}$.

For $x=\pi(g)$ in $X_{\mathbb{Q}} \simeq X_{\mathbb{A}_{f}}$, we define the support of $x$ or support of $g$ :

$$
\operatorname{supp}(x)=\operatorname{supp}(g):=\left\{p \in P \mid x_{p} \neq \pi_{p}(e)\right\} .
$$

Note that $\operatorname{supp}(g)=\left\{p \in P \mid g \notin U_{p}\right\}$ for $g \in \mathbf{G}(\mathbb{Q})$. We say that two elements $g$, $h$ of $\mathbf{G}_{\mathbb{A}_{f}}$ are relatively prime or that their images $x=\pi(g)$ and $y=\pi(h)$ in $X_{\mathbb{A}_{f}}$ are relatively primes if the $\operatorname{supports} \operatorname{supp}(x)$ and $\operatorname{supp}(y)$ are disjoint.

We define the dot-product

$$
z:=x \cdot y
$$

of two relatively prime points of $X_{\mathbb{Q}}$ as the point $z \in X_{\mathbb{Q}}$ such that $z_{p}=x_{p}$ for each $p \in \operatorname{supp}(x), z_{p}=y_{p}$ for each $p \in \operatorname{supp}(y)$ and $x_{p}=x_{0}$ otherwise.

Remark The dot product $z=x . y$ of two points $x=\pi(g)$ and $y=\pi(h)$ is not always equal to $\pi(g h)$. However, if one chooses the lift $h$ of $y$ such that, for all $p$ in $\operatorname{supp}(x)$, $h_{p}$ stabilizes $x_{p}$, then one has $x . y=\pi(g h)$.

The following well known lemma is a main tool for the commutativity of relatively prime Hecke operators.

Lemma 3.10. Let $g$ and $h$ be two relatively prime elements of $\mathbf{G}_{\mathbb{Q}}$.

a) The map $\left(x^{\prime}, y^{\prime}\right) \mapsto x^{\prime} \cdot y^{\prime}$ defines a bijection between $T_{g}\left(x_{0}\right) \times T_{h}\left(x_{0}\right)$ and $T_{g h}\left(x_{0}\right)$.

b) One has $\operatorname{deg}(g h)=\operatorname{deg}(g) \operatorname{deg}(h)$ and

$$
\Gamma g h \Gamma=\Gamma g \Gamma h \Gamma=\Gamma h \Gamma g \Gamma=\Gamma h g \Gamma .
$$

c) One has the equality

$$
\mathrm{T}_{g h}\left(x_{0}\right)=\mathrm{T}_{h g}\left(x_{0}\right)
$$

d) Write $\Gamma g \Gamma=\bigcup_{1 \leq i \leq \operatorname{deg}(g)} \Gamma g_{i}$ and $\Gamma h \Gamma=\bigcup_{1 \leq j \leq \operatorname{deg}(h)} \Gamma h_{j}$. Then

$$
\Gamma g h \Gamma=\bigcup_{i, j} \Gamma g_{i} h_{j}
$$

Proof a) Note that, by Lemma 3.8, one has

$$
\mathrm{T}_{g}\left(x_{0}\right)=\Gamma \backslash \Gamma g \Gamma \simeq U \backslash U g U \simeq \prod_{p \in \operatorname{supp}(\pi(g))} U_{p} \backslash U_{p} g_{p} U_{p} .
$$

Since $g$ and $h$ are relatively prime, one has $U_{p} g_{p} h_{p} U_{p}=U_{p} g_{p} U_{p}$ for any $p \in \operatorname{supp}(\pi(h))$ and $U_{p} g_{p} h_{p} U_{p}=U_{p} h_{p} U_{p}$ for any $p \in \operatorname{supp}(\pi(g))$.

The claims b), c) and d) can be proved similarly. 
We now explain the behavior of the multiplicities with respect to the decomposition into relatively prime factors.

Lemma 3.11. Let $x$ and $y$ be two relatively prime elements of $X_{\mathbb{Q}}$, and $\Delta$ be a subgroup of $\mathbf{G}_{\mathbb{Q}}$. Then one has

a) $\widehat{m}(x \cdot y, \Delta)=\widehat{m}(x, \Delta) \widehat{m}(y, \Delta)$.

b) $m(x \cdot y, \Delta) \geq \max (m(x, \Delta), m(y, \Delta))$.

c) If $\widehat{m}(x, \Delta)=1$, then $m(x \cdot y, \Delta)=m(y, \Delta)$.

Proof a) This equality is a straightforward consequence of the definition of $\widehat{m}$.

b) By Lemma 3.10 and its remark, one can choose $g, h \in \mathbf{G}_{\mathbb{Q}}$ such that $\pi(g)=x$, $\pi(h)=y$ and $\pi(g h)=\pi(h g)=x \cdot y$. Hence one has

$$
\Gamma h^{-1} \Gamma h \subset \Gamma h^{-1} \Gamma g^{-1} \Gamma g h=\Gamma(g h)^{-1} \Gamma g h .
$$

Therefore

$$
\begin{gathered}
\pi\left(h^{-1} \Gamma h\right) \subset \pi\left((g h)^{-1} \Gamma g h\right) \text { and } \\
\Sigma(y, \Delta) \subset \Sigma(x \cdot y, \Delta) .
\end{gathered}
$$

This proves the inequality $m(y, \Delta) \leq m(x \cdot y, \Delta)$. The other direction is proved in the same way.

c) We keep the notation of b) and introduce some new notations: for $S \subset P$ and $z \in X_{\mathbb{Q}}$, let $z_{S} \in X_{\mathbb{Q}}$ be the uniquely determined element with support $S$ and its $p$-component given by $z_{p}$ for each $p \in S$. Let $S:=\operatorname{supp}(x)$ and $T:=\operatorname{supp}(y)$. One has the implications

$$
z \in \pi\left((h g)^{-1} \Gamma h g\right) \Rightarrow z_{S} \in \pi\left(g^{-1} \Gamma g\right)
$$

and

$$
z \in \Sigma(x \cdot y, \Delta) \Rightarrow z_{S} \in \widehat{\Sigma}(x, \Delta) .
$$

We now suppose that $\widehat{m}(x, \Delta)=1$. By the above implication, any element $z \in$ $\Sigma(x \cdot y, \Delta)$ satisfies $\operatorname{supp}(z) \cap S=\emptyset$. Hence, one has successively that $\operatorname{supp}(z) \subset T$, $z \in \pi\left(h^{-1} \Gamma h\right)$ and $z \in \Sigma(y, \Delta)$. This proves

$$
\Sigma(y, \Delta)=\Sigma(x \cdot y, \Delta) .
$$

\section{Bounded MULTiPlicity}

A commutative subgroup $\Delta$ of $\mathbf{G}$ is said to be reductive if all its elements are semisimple.

In this section, we give, for such $\Delta$, an upper bound for the multiplicity of any point $x \in X_{\mathbb{Q}}$ depending only on $\#(\operatorname{supp}(x))$. 


\subsection{Local bound.}

Proposition 4.1. Let $\mathbf{k}$ be a finite extension of $\mathbb{Q}_{p}, \mathbf{G}$ a connected semisimple $\mathbf{k}$-group and $\Delta \subset \mathbf{G}_{\mathbf{k}}$ a commutative reductive subgroup.

There exists an open subgroup $U_{0} \subset \mathbf{G}_{\mathbf{k}}$ such that, for every compact open subgroup $U \subset U_{0}$, there exists a constant $C=C(U, \Delta)$ such that

$$
\text { for any } x \in U \backslash \mathbf{G}_{\mathbf{k}} \text { one has } m_{p}(x, \Delta) \leq C \text {. }
$$

Note that, in view of the restriction of scalars, $\mathbf{G}_{\mathbf{k}}$ is also the group of $\mathbb{Q}_{p}$-points of some semisimple $\mathbb{Q}_{p}$-group $\mathbf{R}_{\mathbf{k} \mid \mathbb{Q}_{p}}(\mathbf{G})$. Hence this proposition is equivalent to the same statement with $\mathbf{k}=\mathbb{Q}_{p}$.

We fix a $\mathbf{k}$-embedding $\mathbf{G} \subset \mathbf{S L}_{\mathbf{N}}$. Let $\mathcal{O}$ be the ring of integers of $\mathbf{k}, \mathcal{M}=\varpi \mathcal{O}$ its maximal ideal, $q:=\#(\mathcal{O} / \mathcal{M})$ and $|$.$| the absolute value on \mathbf{k}$ such that $|\varpi|=q^{-1}$. Let $\mathbf{k}^{\prime}$ be a finite extension of $\mathbf{k}$ which splits all the matrices in $\Delta, \mathcal{O}^{\prime}$ be the ring of integer of $\mathbf{k}^{\prime}$ and choose $g_{\Delta} \in \mathbf{S L}_{\mathbf{N}}\left(\mathbf{k}^{\prime}\right)$ such that $g_{\Delta} \Delta g_{\Delta}^{-1}$ consists of diagonal matrices.

We will see in the proof that one can take

$$
\begin{gathered}
U_{0}=\mathbf{G}_{\mathcal{O}} \cap g_{\Delta}^{-1} \mathbf{S L}_{\mathbf{N}}\left(\mathcal{O}^{\prime}\right) g_{\Delta} \text { and } \\
C(U, \Delta)=N ! C_{U} C_{\Delta} \text { with } \\
C_{U}:=\left[\mathbf{G}_{\mathcal{O}}: U\right] \text { and } C_{\Delta}:=\left[\mathbf{S L}_{\mathbf{N}}\left(\mathcal{O}^{\prime}\right): \mathbf{S L}_{\mathbf{N}}\left(\mathcal{O}^{\prime}\right) \cap g_{\Delta} \mathbf{S L}_{\mathbf{N}}\left(\mathcal{O}^{\prime}\right) g_{\Delta}^{-1}\right]
\end{gathered}
$$

Proof The crucial step is the following important example.

$\mathbf{1}^{\text {st }}$ case : $\mathbf{G}=\mathbf{P G L}_{\mathbf{N}}, U=\mathbf{P G L}_{\mathbf{N}}(\mathcal{O})$ and $\Delta=\left\{\right.$ diagonal matrices of $\left.\mathbf{G}_{\mathbf{k}}\right\}$.

We can identify the homogeneous space $U \backslash \mathbf{G}_{\mathbf{k}}$ with the space of lattices in $\mathbf{k}^{N}$, i.e. of $\mathcal{O}$-submodules of rank $N$, up to homothety by the multiplicative group $\mathbf{k}^{*}$.

The point $x_{0}$ corresponds to the lattice $\Lambda_{0}:=\mathcal{O}^{N}$ and the point $x=x_{0} g$ corresponds to the lattice $\Lambda=g^{-1}\left(\mathcal{O}^{N}\right)$ up to homothety. We may and will choose this lattice $\Lambda$ to be a primitive sublattice of $\Lambda_{0}$ i.e. $\Lambda \subset \Lambda_{0}$ and $\Lambda \not \subset \varpi \Lambda_{0}$. Let $e_{1}, \ldots, e_{N}$ be the standard basis of the $\mathcal{O}$-module $\Lambda_{0}=\mathcal{O}^{N}$. Since $\mathcal{O}$ is principal, there exists integers $0=m_{1} \leq \cdots \leq m_{N}$ and a basis $f_{1}, \ldots, f_{N}$ of $\Lambda_{0}$ such that

$$
\Lambda=\mathcal{O} \varpi^{m_{1}} f_{1} \oplus \cdots \oplus \mathcal{O} \varpi^{m_{N}} f_{N}
$$

Set

$$
a:=\operatorname{diag}\left(\varpi^{-m_{1}}, \ldots, \varpi^{-m_{N}}\right) \in \mathbf{P G L}_{\mathbf{N}}(\mathbf{k}) .
$$

Then one has $x \in \mathrm{T}_{a}\left(x_{0}\right)$. We will write equivalently $\Lambda \in \mathrm{T}_{a}\left(\Lambda_{0}\right)$. Recalling the definition of $\Sigma_{p}(x, \Delta)$, one sees that

$$
\Sigma_{p}(x, \Delta) \simeq\left\{\Lambda_{d}:=\mathcal{O} \varpi^{d_{1}} e_{1} \oplus \cdots \oplus \mathcal{O} \varpi^{d_{N}} e_{N} \mid d \in \mathbb{Z}^{N}, \Lambda \in \mathrm{T}_{a}\left(\Lambda_{d}\right)\right\} / \mathbf{k}^{*} .
$$

Any element of $\Sigma_{p}(x, \Delta)$ has a unique representative $\Lambda_{d}$ which contains $\Lambda$ as a primitive submodule. This submodule $\Lambda_{d}$ has same volume as $\Lambda_{0}$. This avoids the homothety 
by $\mathbf{k}^{*}$ and gives the identification

$$
\Sigma_{p}(x, \Delta) \simeq\left\{\Lambda_{d}:=\mathcal{O} \varpi^{d_{1}} e_{1} \oplus \cdots \oplus \mathcal{O} \varpi^{d_{N}} e_{N} \mid d \in \mathbb{Z}^{N}, \Lambda \in \mathrm{T}_{a}\left(\Lambda_{d}\right), \sum d_{i}=0\right\} .
$$

Note that this set $\Sigma_{p}(x, \Delta)$ does not depend on the choice of $\Lambda_{0}$, provided $\Lambda_{0}$ is in $\mathrm{T}_{a^{-1}}(\Lambda)$ and $\Lambda_{0}$ admits a $\mathcal{O}$-basis with respect to which $\Delta$ is diagonal. Hence we are allowed to replace $\Lambda_{0}$ by any of the $\Lambda_{d} \in \Sigma_{p}(x, \Delta)$. We will do that later on.

To prove the desired inequality

$$
\#\left(\Sigma_{p}(x, \Delta)\right) \leq N \text { ! }
$$

we will argue by induction on $N$.

Define for all $i$ the integers $n_{i}$ by the equalities $\mathbf{k} e_{i} \cap \Lambda=\mathcal{O} \varpi^{n_{i}} e_{i}$. By (4.3), they satisfy the following inequalities

for any $i, \quad m_{1} \leq n_{i} \leq m_{N}$ and there exists $k_{0}$ such that $n_{k_{0}}=m_{N}$.

For the same reason, using the equalities $\mathbf{k} \varpi^{d_{i}} e_{i} \cap \Lambda=\mathcal{O} \varpi^{n_{i}-d_{i}}\left(\varpi^{d_{i}} e_{i}\right)$, one gets the inequalities, for any $\Lambda_{d} \in \Sigma_{p}(x, \Delta)$,

for any $i, m_{1} \leq n_{i}-d_{i} \leq m_{N}$ and there exists $i_{0}$ such that $n_{i_{0}}-d_{i_{0}}=m_{N}$.

Fixing $i_{0} \in\{1, \ldots, N\}$, it is enough to show that the set

$$
S_{i_{0}}:=\left\{\Lambda_{d} \in \Sigma_{p}(x, \Delta) \mid n_{i_{0}}-d_{i_{0}}=m_{N}\right\}
$$

has at most $(N-1)$ ! elements. Replacing $\Lambda_{0}$ by any element $\Lambda_{d}$ of $S_{i_{0}}$ as explained above, one can assume that $\Lambda_{0}$ belongs to $S_{i_{0}}$ and hence $k_{0}=i_{0}$. Therefore one has

$$
n_{i_{0}}=m_{N} \quad \text { and } \quad \text { for any } \Lambda_{d} \in S_{i_{0}} \quad d_{i_{0}}=0 .
$$

Let $p_{i_{0}}: \mathbf{k}^{N} \rightarrow \mathbf{k}^{N-1}$ be the projection which forgets the $i_{0}^{\text {th }}$ coordinate, and set

$$
\Lambda_{0}^{\prime}:=p_{i_{0}}\left(\Lambda_{0}\right), \Lambda^{\prime}:=p_{i_{0}}(\Lambda), \Lambda_{d}^{\prime}:=p_{i_{0}}\left(\Lambda_{d}\right)
$$

and $a^{\prime}=\operatorname{diag}\left(\varpi^{-m_{1}}, \ldots, \varpi^{-m_{N-1}}\right)$. One has the following exact sequence of finite $\mathcal{O}$-modules

$$
0 \longrightarrow \mathcal{O} /\left(\mathcal{O} \omega^{n_{i_{0}}}\right) \longrightarrow \Lambda_{0} / \Lambda \longrightarrow \Lambda_{0}^{\prime} / \Lambda^{\prime} \longrightarrow 0 \text {. }
$$

Since, by (4.4), one has $n_{i_{0}}=m_{N}=\max \left(m_{i}\right)$, this sequence splits and $\Lambda^{\prime}$ is contained in $T_{a^{\prime}}\left(\Lambda_{0}^{\prime}\right)$. Similarly,

$$
\Lambda^{\prime} \in T_{a^{\prime}}\left(\Lambda_{d}^{\prime}\right) \quad \text { for all } \Lambda_{d} \in S_{i_{0}} .
$$

Hence by induction hypothesis, there are at most $(N-1)$ ! such $\Lambda_{d}^{\prime}$. This concludes the proof of this example.

$2^{\text {nd }}$ case : We now reduce the proof of the general case to the above example.

Using the chosen $\mathbf{k}$-embedding $\mathbf{G} \subset \mathbf{S L}_{\mathbf{N}}$, set

$$
U_{0}:=\mathbf{G}_{\mathcal{O}} \cap g_{\Delta}^{-1} \mathbf{S L}_{\mathbf{N}}\left(\mathcal{O}^{\prime}\right) g_{\Delta} .
$$

Using Lemma 3.4.a with $U^{\prime}=U_{0}$, we can suppose that $U=U^{\prime}$. This gives the factor $C_{U}$ in the constant $C$. 
Using Lemma 3.4.c with $G^{\prime}=\mathbf{S L}_{\mathbf{N}}$, and changing the base field $\mathbf{k}$ by $\mathbf{k}^{\prime}$, we can suppose that $G=\mathbf{S L}_{\mathbf{N}}, \mathbf{k}^{\prime}=\mathbf{k}$ and $U=\mathbf{G}_{\mathcal{O}} \cap g^{-1} \mathbf{G}_{\mathcal{O}} g$. This does not change the constant $C$.

Using again Lemma 3.4.a with $U^{\prime}=g^{-1} \mathbf{G}_{\mathcal{O}} g$ we can suppose moreover that $g=e$. This gives the factor $C_{\Delta}$ in the constant $C$.

Using Lemma 3.4.b with $\Delta^{\prime}=\{$ diagonal matrices $\}$, we can suppose that $\Delta=\Delta^{\prime}$ This does not change the constant $C$.

Hence we have $\mathbf{G}=\mathbf{S L}_{\mathbf{N}}, U=\mathbf{S L}_{\mathbf{N}}(\mathcal{O})$ and $\Delta=\{$ diagonal matrices $\}$ and our result is a consequence of the first case.

4.2. Independence of $p$. The following proposition is a global version of Proposition 4.1.

For $x=\pi(g) \in X_{\mathbb{Q}}$, let

$$
s(g)=s(x):=\#(\operatorname{supp}(x))
$$

(roughly, this integer $s(g)$ can be thought as the number of primes which appear in the denominators of the coefficients of the matrix $g$ ).

Proposition 4.5. Let $\mathbf{G}$ be a connected almost $\mathbb{Q}$-simple $\mathbb{Q}$-group. Let $\Delta \subset \mathbf{G}_{\mathbb{Q}} a$ commutative reductive subgroup.

There exists a congruence subgroup $\Gamma_{0} \subset \mathbf{G}_{\mathbb{Q}}$ such that, for every congruence subgroup $\Gamma \subset \Gamma_{0}$ of the form $\Gamma=G_{\mathbb{Q}} \cap \prod_{p} U_{p}$ for compact open subgroups $U_{p} \subset \mathbf{G}_{\mathbb{Q}_{p}}$, there exists a constant $C=C(\Gamma, \Delta)$ such that, for each $x \in X_{\mathbb{Q}}$, one has

$$
m(x, \Delta) \leq C^{s(x)} \text {. }
$$

Proof We fix a $\mathbb{Q}$-embedding $\mathbf{G} \subset \mathbf{S L}_{\mathbf{N}}$. By Proposition 4.1, for each prime $p$, there exists a compact open subgroup $U_{0}(p)$ of $\mathbf{G}_{\mathbb{Q}_{p}}$ such that, for every compact open subgroup $V_{p} \subset U_{0}(p)$, there exists a constant $C=C\left(V_{p}, \Delta\right)$ such that

$$
\text { for any } x \in V_{p} \backslash \mathbf{G}_{\mathbb{Q}_{p}} \text { one has } m_{p}(x, \Delta) \leq C \text {. }
$$

Since one can diagonalize $\Delta$ on a finite extension of $\mathbb{Q}$, it follows from (4.2) that we can suppose $U_{0}(p)=\mathbf{G}_{\mathbb{Z}_{p}}$ for almost all $p$. More precisely, let us choose a finite extension $L$ of $\mathbb{Q}$ and an element $g_{\Delta} \in \mathbf{S L}_{\mathbf{N}}(L)$ such that $g_{\Delta} \Delta g_{\Delta}^{-1}$ consist of diagonal matrices. For any $p$ choose a finite extension $\mathbf{k}^{\prime}$ of $\mathbb{Q}_{p}$ containing an embedding of $L$. The formula (4.2) gives $U_{0}(p)=\mathbf{G}_{\mathcal{O}} \cap g_{\Delta}^{-1} \mathbf{S L}_{\mathbf{N}}\left(\mathcal{O}^{\prime}\right) g_{\Delta}$. But for almost all $p$ the element $g_{\Delta}$ is in $\mathbf{S L}_{\mathbf{N}}\left(\mathcal{O}^{\prime}\right)$, hence one has $U_{0}(p)=\mathbf{G}_{\mathbb{Z}_{p}}$.

Now if we set $\Gamma_{0}:=G_{\mathbb{Q}} \cap \prod_{p} U_{0}(p), \Gamma_{0}$ is a congruence subgroup. Since $U_{p}=\mathbf{G}_{\mathbb{Z}_{p}}$ for almost all $p, \Gamma$ being a congruence subgroup, it follows from $(4.2)$ that $C\left(U_{p}, \Delta\right)=N$ ! for almost all $p$. Therefore there exists $C=C(\Gamma, \Delta)$ such that

$$
m_{p}(x, \Delta) \leq C
$$

for all $x \in X_{\mathbb{Q}}$ and for all $p \in P$.

Since $m_{p}(x)=1$, whenever $p$ is not in the support of $x$, the claim now follows from Lemma 3.7. 


\section{Equidistribution for Finitely MANy PRIMES With $\Gamma$ GENERAL}

5.1. Ergodicity argument. Let $G, \Gamma, X$ and $H, \mu_{a}=\mu_{a}^{H}$ be as in Section 2. Let $x_{0} H \subset X$ be an $H$-orbit with finite volume.

Lemma 5.1. Let $S$ be a sequence of elements a in $G_{\mathbb{Q}}$ with $\operatorname{deg}(a) \rightarrow \infty$. Suppose that $H$ is non-compact and that

$$
\sup _{a \in S}\left(\max _{x_{i} \in \mathrm{T}_{a}\left(x_{0}\right)} m\left(x_{i}, H\right)\right)<\infty .
$$

Then one has $\lim _{a \in S} \mu_{a}^{H}=\mu_{X}$.

Proof Let $\mathcal{P}(\bar{X})$ be the space of probability measures on the one-point compactification $\bar{X}:=X \cup\{\infty\}$ when $X$ is non compact and on $\bar{X}:=X$ when $X$ is compact. By the weak compactness of this space $\mathcal{P}(\bar{X})$, it is enough to show that each weak limit of a subsequence of $\mu_{a}$ is equal to $\mu_{X}$. Let $\mu_{\infty}$ be such a weak limit.

Let $m_{0} \geq 2$ be an upper bound for all the multiplicities $m\left(x_{i}\right)$. One has, for all $a$ in $S$,

$$
\frac{1}{m_{0}} \operatorname{deg}(a) \leq \widetilde{\operatorname{deg}}(a)
$$

and if $f \in \mathrm{C}_{c}(X)$ is a non-negative function,

$$
\widetilde{\mathrm{T}}_{a} f \leq m_{0} \mathrm{~T}_{a} f .
$$

Applying Theorem 2.1 and Lemma 2.3.c, by integrating the previous inequality on $Y$, one gets for the limit measures in $\mathcal{P}(\bar{X})$

$$
\mu_{\infty} \leq m_{0} \mu_{X}
$$

In particular, the probability measure $\mu_{\infty}$ is supported on $X$. Since $H$ is non-compact, Moore's ergodicity theorem (cf. [Zi]) tells us that the action of $H$ on $X$ is ergodic for $\mu_{X}$. Hence $\mu_{X}$ is an extremal point of the set of $H$-invariant probability measures on $X$. The equality

$$
\mu_{X}=\left(1-\frac{1}{m_{0}}\right)\left(\frac{m_{0} \mu_{X}-\mu_{\infty}}{m_{0}-1}\right)+\frac{1}{m_{0}} \mu_{\infty}
$$

implies then $\mu_{\infty}=\mu_{X}$.

\subsection{Changing the congruence subgroup.}

Lemma 5.3. It suffices to prove Theorem 1.1 for a subgroup $\Gamma^{\prime}$ of $\Gamma$ of finite index.

Proof The main idea is to use the ergodicity argument of the proof of Lemma 5.1. First, let us adapt the notations we have already introduced for the group $\Gamma$ to the group $\Gamma^{\prime}$ by adding primes: set

$$
X^{\prime}:=\Gamma^{\prime} \backslash G, \pi^{\prime}: G \rightarrow X^{\prime}, x_{0}^{\prime}:=\pi^{\prime}\left(g_{0}\right), Y^{\prime}:=x_{0}^{\prime} H, T_{a}^{\prime}\left(Y^{\prime}\right):=\pi^{\prime}\left(a \pi^{\prime-1}\left(Y^{\prime}\right)\right) .
$$


Let $\mu_{X^{\prime}}$ denote the $G$-invariant probability measure on $X^{\prime}$, and $\lambda_{a}^{\prime}$ the measure on $T_{a}^{\prime}\left(Y^{\prime}\right)$ which is locally equal to $\lambda_{H}$. Also set

$$
\widetilde{\operatorname{deg}^{\prime}} a:=\lambda_{a}^{\prime}\left(X^{\prime}\right) \quad \text { and } \quad \mu_{a}^{\prime}:=\left(\widetilde{\operatorname{deg}^{\prime} a}\right)^{-1} \lambda_{a}^{\prime}
$$

Write $\Gamma=\bigcup_{1 \leq j \leq d} g_{j} \Gamma^{\prime}$ where $d=\left[\Gamma: \Gamma^{\prime}\right]$ is the degree of the covering $p: X^{\prime} \rightarrow X$. Notice that the measures $p_{*}\left(\lambda_{a g_{j}}^{\prime}\right)$ are locally equal to an integral multiple $m \lambda_{H}$ of $\lambda_{H}$, for some $m \in\{1, \cdots, d\}$ on each of the finitely many $H$-orbits in $p\left(T_{a g_{j}}^{\prime}\left(Y^{\prime}\right)\right)$. As a consequence, one has the inequality

$$
\lambda_{a} \leq \sum_{1 \leq j \leq d} p_{*}\left(\lambda_{a g_{j}}^{\prime}\right)
$$

Since, for all $j, \frac{1}{d} p_{*}\left(\lambda_{a g_{j}}^{\prime}\right)(X) \leq \lambda_{a}(X)$, one has the inequality

$$
\mu_{a} \leq d \sum_{1 \leq j \leq d} p_{*}\left(\mu_{a g_{j}}^{\prime}\right)
$$

By hypothesis, for each $1 \leq j \leq d$, one has

$$
\lim _{a \in S} \mu_{a g_{j}}^{\prime}=\mu_{X^{\prime}}
$$

Hence any weak limit $\mu_{\infty}$ of the sequence $\mu_{a}$ satisfies

$$
\mu_{\infty} \leq d^{2} \mu_{X}
$$

Such a weak limit is an $H$-invariant probability measure supported on $X$ and absolutely continuous with respect to $\mu_{X}$. Since $\mu_{X}$ is an ergodic measure for the action of $H$, it follows $\mu_{\infty}=\mu_{X}$. This proves that $\lim _{a \in S} \mu_{a}=\mu_{X}$.

Remark 5.4. Note that in the above proof we have not used any of the assumption $\mathbf{G}$ simply connected, $\Gamma$ cocompact or $H$ commutative reductive.

5.3. Proof of Theorem $\mathbf{1 . 1}$ for $\# \operatorname{supp}(a)$ uniformly bounded. We now prove Theorem 1.1 in the case when $\# \operatorname{supp}(a)$ is uniformly bounded. Note that we do not need $\mathbf{G}$ simply connected or $\Gamma$ cocompact in this case.

Theorem 5.5. Let $\mathbf{G}$ be a connected almost $\mathbb{Q}$-simple algebraic $\mathbb{Q}$-group with $G=$ $\mathbf{G}(\mathbb{R})^{\circ}$ and $H$ a closed commutative reductive non-compact subgroup of $G$. Let $\Gamma$ be any congruence subgroup in $G_{\mathbb{Q}}$ and $x_{0} H \subset X$ an $H$-orbit with finite volume. Let $S$ be a sequence of elements $a \in G_{\mathbb{Q}}$ such that \# $\operatorname{supp}(a)$ is uniformly bounded for all $a \in S$ and $\operatorname{deg}(a) \rightarrow \infty$. Then for any $f \in \mathrm{C}_{c}(X)$, one has

$$
\lim _{a \in S} \frac{1}{\lambda_{a}^{H}(X)} \int_{X} f d \lambda_{a}^{H}=\int_{X} f d \mu_{X} .
$$


Proof One can easily reduce our proofs to the case when $x_{0}=\pi(e)$ : simply by replacing the group $H$ by $g_{0}^{-1} H g_{0}$ for $x_{0}=\pi\left(g_{0}\right)$. Let $\Gamma_{0}$ be a congruence subgroup as in Proposition 4.5 with respect to $\Delta=H \cap \mathbf{G}_{\mathbb{Q}}$. In view of Lemma 5.3 and Remark 5.4, there is no loss of generality in assuming that $\Gamma \subset \Gamma_{0}$ and $\Gamma$ is of the form $G_{\mathbb{Q}} \cap \prod_{p} U_{p}$ by replacing $\Gamma$ by a finite index subgroup if necessary. Since $m\left(x_{i}, H\right)=m\left(x_{i}, \Delta\right)$ by the remark following Definition 3.1 and $\operatorname{supp}(a)=\operatorname{supp}\left(x_{i}\right)$ for any $x_{i} \in \mathrm{T}_{a}\left(x_{0}\right)$, Proposition 4.5 implies that the multiplicities $m\left(x_{i}\right)$ are uniformly bounded for all $\cup_{a \in S} \mathrm{~T}_{a}\left(x_{0}\right)$. Hence it only remains to apply Lemma 5.1, to finish the proof.

\section{HeCKE POINTS With LOCAL MULTiplicity ONE}

For $a \in \mathbf{G}_{\mathbb{Q}}, p$ prime, and a subgroup $\Delta$ of $\mathbf{G}_{\mathbb{Q}}$, set

$$
S_{a}\left(x_{0}, \Delta, p\right):=\left\{x \in \mathrm{T}_{a}\left(x_{0}\right) \mid m_{p}(x, \Delta)=1\right\}
$$

which is the set of Hecke points with p-multiplicity one.

The entire section is devoted to a proof of the following theorem that most Hecke points have $p$-multiplicity one for all $p$ large.

Theorem 6.1. Let $\mathbf{G}$ be a connected simply connected almost $\mathbb{Q}$-simple $\mathbb{Q}$-group with $\mathbf{G}_{\mathbb{R}}$ non-compact and $\Gamma$ a congruence subgroup of the form $\Gamma=\mathbf{G}_{\mathbb{Q}} \cap \prod_{p} U_{p}$ for compact open subgroups $U_{p} \subset \mathbf{G}_{\mathbb{Q}_{p}}$. Let $\Delta \subset \mathbf{G}_{\mathbb{Q}}$ be a commutative reductive subgroup.

Then there exists a constant $D=D(\Gamma, \Delta)>0$ such that, for all primes $p$ and all $a \in \mathbf{G}_{\mathbb{Q}}$, one has

$$
\#\left(S_{a}\left(x_{0}, \Delta, p\right)\right) \geq\left(1-\frac{D}{p}\right) \#\left(\mathrm{~T}_{a}\left(x_{0}\right)\right) .
$$

\subsection{Multiplicities and modules.}

Lemma 6.2. Let $\mathbf{H} \subset \mathbf{S L}_{\mathbf{N}}$ be a $\mathbb{Q}$-torus. Then, for almost all prime $p \in P$, there exist $N \mathbb{Z}_{p}$-submodules $\Lambda_{1}, \ldots, \Lambda_{N}$ of $\Lambda_{0}:=\left(\mathbb{Z}_{p}\right)^{N}$ of index $p$ such that, for any $h \in \mathbf{H}_{\mathbb{Q}_{p}}$ with $h \notin \mathbf{H}_{\mathbb{Z}_{p}}$, one has

$$
\Lambda_{0} \cap h\left(\Lambda_{0}\right) \subset \Lambda_{i} \quad \text { for some } i \in\{1, \cdots, N\} .
$$

Proof We first begin by a very simple example.

$\mathbf{1}^{\text {st }}$ case : $\mathbf{H}=\left\{\right.$ diagonal matrices of $\left.\mathbf{S L}_{\mathbf{N}}\right\}$.

In this case, one can take

$$
\Lambda_{i}:=\left\{\left(x_{1}, \ldots, x_{N}\right) \in \mathbb{Z}_{p}^{N} \mid x_{i} \equiv 0 \bmod p\right\} .
$$

Let $h \in \mathbf{H}_{\mathbb{Q}_{p}}$. Write

$$
h=\operatorname{diag}\left(p^{d_{1}} u_{1}, \ldots, p^{d_{N}} u_{N}\right),
$$

where $u_{i}$ are units in $\mathbb{Z}_{p}$. Note that, since $\operatorname{det}(h)=1$, one has $d_{1}+\cdots+d_{N}=0$. Moreover, if $h \notin \mathbf{H}_{\mathbb{Z}_{p}}$, at least one of the $d_{i}$ is negative. Hence one of the $d_{i}$, say $d_{i_{0}}$ is positive. It follows that

$$
\Lambda_{0} \cap h\left(\Lambda_{0}\right) \subset \Lambda_{i_{0}}
$$


General case The proof is along the same lines as in the first case except that we will have to change the base field to diagonalize the torus. Here are the details.

Let $E$ be a number field on which the torus $\mathbf{H}$ splits and $g \in \mathbf{G L}_{\mathbf{N}}(E)$ a matrix for which $g \mathbf{H} g^{-1}$ is diagonal. We choose for each prime $p$ a valuation $v$ of $E$ over $p$ and let $E_{v} \supset \mathbb{Q}_{p}$ be the completion of $E, \mathcal{O}_{v}$ the ring of integers of $E_{v}, \mathcal{M}_{v}$ the maximal ideal of $\mathcal{O}_{v}$ and $\varpi$ a uniformizer in $\mathcal{M}_{v}$. For almost all primes $p$, the matrix $g$ belongs to $\mathbf{S L}_{\mathbf{N}}\left(\mathcal{O}_{v}\right)$. In this case, one can take for $\Lambda_{i}$ any index $p$ submodule of $\mathbb{Z}_{p}^{N}$ such that

$$
\Lambda_{i} \supset \mathbb{Z}_{p}^{N} \cap g^{-1}\left(M_{i, v}\right) \quad \text { where } M_{i, v}:=\left\{\left(x_{1}, \ldots, x_{N}\right) \in \mathcal{O}_{v}^{N} \mid x_{i} \in \mathcal{M}_{v}\right\} .
$$

Note that, since $g\left(\mathcal{O}_{v}^{N}\right)=\mathcal{O}_{v}^{N}$, the intersection $\mathbb{Z}_{p}^{N} \cap g^{-1}\left(M_{i, v}\right)$ is not equal to $\mathbb{Z}_{p}^{N}$. Let $h \in \mathbf{H}_{\mathbb{Q}_{p}}$ and $h \notin \mathbf{H}_{\mathbb{Z}_{p}}$. One can write

$$
g h g^{-1}=\operatorname{diag}\left(\varpi^{d_{1}} u_{1}, \ldots, \varpi^{d_{N}} u_{N}\right),
$$

where $u_{i}$ are units in $\mathcal{O}_{v}$. Note that, since $\operatorname{det}(h)=1$, one has $d_{1}+\cdots+d_{N}=0$. Moreover, since $h \notin \mathbf{H}_{\mathcal{O}_{v}}$, at least one of the $d_{i}$ is negative. Hence one of the $d_{i}$, say $d_{i_{0}}$ is positive. This implies

$$
\Lambda_{0} \cap h\left(\Lambda_{0}\right) \subset \Lambda_{i_{0}}
$$

Proposition 6.3. Let $\mathbf{G} \subset \mathbf{S L}_{\mathbf{N}}$ be a connected semisimple $\mathbb{Q}$-group, $\Gamma \subset \mathbf{G}_{\mathbb{Q}} a$ congruence subgroup of the form $\Gamma=\mathbf{G}_{\mathbb{Q}} \cap \prod_{p} U_{p}$ for compact open subgroups $U_{p} \subset \mathbf{G}_{\mathbb{Q}_{p}}$, $\mathbf{H} \subset \mathbf{G}$ a $\mathbb{Q}$-torus and $\Delta$ a subgroup of $\mathbf{H}_{\mathbb{Q}}$.

Then, for almost all primes $p$, there exist $N \mathbb{Z}_{p}$-submodules $\Lambda_{1}, \ldots, \Lambda_{N}$ of $\Lambda_{0}:=$ $\left(\mathbb{Z}_{p}\right)^{N}$ of index $p$ such that, for all $a \in \mathbf{G}_{\mathbb{Q}_{p}}$, for all $\Lambda \in \mathrm{T}_{a}\left(\Lambda_{0}\right)$, one has the implication

$$
m_{p}(\Lambda, \Delta)>1 \Longrightarrow \Lambda \subset \Lambda_{i} \text { for some } i \in\{1, \cdots, N\} \text {. }
$$

In the above, we have identified, for almost all $p$, the homogeneous space $U_{p} \backslash \mathbf{G}_{\mathbb{Q}_{p}}$ with a subspace of the space $\mathbf{P G} \mathbf{L}_{\mathbf{N}}\left(\mathbb{Z}_{p}\right) \backslash \mathbf{P G L}_{\mathbf{N}}\left(\mathbb{Q}_{p}\right)$. Note that the latter space can be identified with the set of $\mathbb{Z}_{p}$-submodules of $\mathbb{Q}_{p}^{N}$ of rank $N$ up to homothety by $\mathbb{Q}_{p}^{*}$. Since $\mathbb{Z}_{p}$ is principal, we may further identify $\mathbf{P G L}_{\mathbf{N}}\left(\mathbb{Z}_{p}\right) \backslash \mathbf{P G L}_{\mathbf{N}}\left(\mathbb{Q}_{p}\right)$ with the space of $\mathbb{Z}_{p}$-submodules $\Lambda$ of finite index in $\Lambda_{0}$ which are primitive, i.e. such that $\Lambda \not \subset p \Lambda_{0}$. If $a=\operatorname{diag}\left(p^{-m_{1}}, \ldots, p^{-m_{N}}\right)$ with $m_{1} \leq \cdots \leq m_{N}$, one has the equivalence

$$
\Lambda \in \mathrm{T}_{a}\left(\Lambda_{0}\right) \Longleftrightarrow \Lambda_{0} / \Lambda \simeq\left(\mathbb{Z} / p^{m_{2}-m_{1}} \mathbb{Z}\right) \times \cdots \times\left(\mathbb{Z} / p^{m_{N}-m_{1}} \mathbb{Z}\right) .
$$

Proof of Proposition 6.3 By Lemma 3.4.c, one can assume that $\mathbf{G}=\mathbf{S L}_{\mathbf{N}}$. Since $m_{p}(\Lambda, \Delta)>1$, there exists $h \in \mathbf{H}_{\mathbb{Q}_{p}}$ such that

$$
h\left(\Lambda_{0}\right) \neq \Lambda_{0} \quad \text { and } \quad \Lambda \in \mathrm{T}_{a}\left(h\left(\Lambda_{0}\right)\right) .
$$

Choose a scalar $k \in \mathbb{Q}_{p}^{*}$ such that the $\mathbb{Z}_{p}$-module $k \Lambda$ is a primitive submodule of $h\left(\Lambda_{0}\right)$. One has

$$
\left[h\left(\Lambda_{0}\right): k \Lambda\right]=\left[\Lambda_{0}: \Lambda\right]
$$


Since the $\mathbb{Z}_{p}$-modules $\Lambda_{0}$ and $h\left(\Lambda_{0}\right)$ have same volumes, it follows that the $\mathbb{Z}_{p}$-modules $\Lambda$ and $k \Lambda$ have same volumes and hence $\Lambda=k \Lambda$. Therefore one gets the inclusion

$$
\Lambda \subset \Lambda_{0} \cap h\left(\Lambda_{0}\right)
$$

It only remains to apply Lemma 6.2.

6.2. Counting Hecke points. We will also need the following lemma in the proof of Theorem 6.1.

Lemma 6.4. Let $\mathbf{G} \subset \mathbf{S L}_{\mathbf{N}}$ be a semisimple $\mathbb{Q}$-group. We suppose that this $\mathbb{Q}$ embedding of $\mathbf{G}$ is an absolutely irreducible $\mathbb{Q}$-representation. Then there exists a constant $D_{0}>0$, such that, for almost all primes $p$, all $a \in \mathbf{G}_{\mathbb{Q}_{p}}$ and all submodule $\Lambda_{1}$ of $\Lambda_{0}:=\left(\mathbb{Z}_{p}\right)^{N}$ of index $p$, one has

$$
\frac{\#\left\{\Lambda \in \mathrm{T}_{a}\left(\Lambda_{0}\right) \mid \Lambda \subset \Lambda_{1}\right\}}{\operatorname{deg}(a)} \leq \frac{D_{0}}{p}
$$

The absolute irreducibility assumption in this lemma means the irreducibility over the algebraic closure, which is stronger than the irreducibility over $\mathbb{Q}$. It will be needed to apply the fact 6.6 .

To prove as well as to apply this lemma 6.4 we will need the following general four facts.

Fact 6.5. Every connected semisimple $\mathbb{Q}$-group $\mathbf{G}$ with trivial center has an absolutely irreducible faithful $\mathbb{Q}$-representation $\rho: \mathbf{G} \rightarrow \mathbf{S L}_{\mathbf{N}}$.

The term $\mathbb{Q}$-representation means representation defined over $\mathbb{Q}$.

Proof Recall that, according to the highest weight theory, the set $R^{+}$of "dominant weights of the root lattice" parameterizes the set of (equivalence classes of absolutely) irreducible representations of G. Since the action of the Galois group $\operatorname{Gal}(\overline{\mathbb{Q}} / \mathbb{Q})$ on $R^{+}$factors through the linear action of the finite group of exterior automorphisms of $\mathbf{G}$, there exist many fixed points for this action.

Hence one can choose a faithful absolutely irreducible representation of $\mathbf{G}$ of highest weight $\lambda$ which is equivalent to all its Galois conjugates. According to [Ti, Theorem 3.3], since $\lambda$ is in the root lattice, this representation is defined over $\mathbb{Q}$.

Fact 6.6. Let $\rho: \mathbf{G} \rightarrow \mathbf{S L}_{\mathbf{N}}$ be an absolutely irreducible $\mathbb{Q}$-representation of a connected semisimple $\mathbb{Q}$-group $\mathbf{G}$. Then for almost all primes $p$, one gets by reduction modulo $p$, a representation $\mathbf{G}_{\mathbb{F}_{p}} \rightarrow \mathbf{S L}_{\mathbf{N}}\left(\mathbb{F}_{p}\right)$ which is still absolutely irreducible.

Proof Let $A_{\mathbb{Q}} \subset \mathcal{M}_{N}(\mathbb{Q})$ be the $\mathbb{Q}$-algebra generated by $\rho\left(\mathbf{G}_{\mathbb{Q}}\right), L \subset \rho\left(\mathbf{G}_{\mathbb{Q}}\right)$ a finitely generated subgroup generating the $\mathbb{Q}$-algebra $A_{\mathbb{Q}}$ and $m$ an integer such that $L \subset \mathcal{M}_{N}\left(\mathbb{Z}\left[\frac{1}{m}\right]\right)$. By Wedderburn's theorem, since the representation is absolutely irreducible, one has $A_{\mathbb{Q}}=\mathcal{M}_{N}(\mathbb{Q})$. Hence there exists an integer $m_{0}$ such that the $\mathbb{Z}\left[\frac{1}{m}\right]$-algebra $A$ generated by $L$ contains $m_{0} \mathcal{M}_{N}\left(\mathbb{Z}\left[\frac{1}{m}\right]\right)$. 
If one chooses $p$ outside the divisors of $m m_{0}$, the image of $L$ in $\mathcal{M}_{N}\left(\mathbb{F}_{p}\right)$ generates $\mathcal{M}_{N}\left(\mathbb{F}_{p}\right)$ and the representation $\mathbf{G}_{\mathbb{F}_{p}} \rightarrow \mathbf{S L}_{\mathbf{N}}\left(\mathbb{F}_{p}\right)$ is absolutely irreducible.

Fact 6.7. Let $\mathbf{G}$ be a connected $\mathbb{F}_{p}$-group and $\mathbf{H}$ a connected $\mathbb{F}_{p}$-subgroup. Then one has the equality

$$
(\mathbf{G} / \mathbf{H})_{\mathbb{F}_{p}}=\mathbf{G}_{\mathbb{F}_{p}} / \mathbf{H}_{\mathbb{F}_{p}}
$$

Proof See Proposition 16.5 of [Bo].

Fact 6.8. For any absolutely irreducible projective $\mathbb{F}_{p}$-variety $\mathbf{M} \subset \mathbb{P}^{N_{0}}$ of dimension $D$ and degree $d$, one has

$$
\left|\#\left(\mathbf{M}_{\mathbb{F}_{p}}\right)-p^{D}\right| \leq A p^{D-\frac{1}{2}}
$$

where the constant $A$ depends only on $N_{0}, D$ and $d$.

Proof This generalization of Hasse's theorem is due to Lang and Weil (see [LW]).

Proof of Lemma 6.4 The Lemma is clear if $a$ is in $\mathbf{G}_{\mathbb{Z}_{p}}$. Hence we may suppose that $a \notin \mathbf{G}_{\mathbb{Z}_{p}}$. We introduce the map $\Phi$ from the Hecke orbit $\mathrm{T}_{a}\left(\Lambda_{0}\right)$ to the Grassmanian $\operatorname{Gr}_{\mathbf{N}}\left(\mathbb{F}_{p}\right)$ of $\mathbb{F}_{p}^{N}$ given by

$$
\Phi(\Lambda):=\left(\Lambda+p \Lambda_{0}\right) / p \Lambda_{0}
$$

Here, as in Proposition $6.3, \Lambda$ is viewed as a primitive $\mathbb{Z}_{p}$-submodule of $\Lambda_{0}$ so that $\Phi(\Lambda) \neq 0$. Moreover, since $a$ is not in $\mathbf{G}_{\mathbb{Z}_{p}}, \Phi(\Lambda) \neq \Lambda_{0} / p \Lambda_{0}$.

This map is $\mathbf{G}_{\mathbb{Z}_{p}}$-equivariant where the action of $\mathbf{G}_{\mathbb{Z}_{p}}$ on $\mathbf{G r}_{\mathbf{N}}\left(\mathbb{F}_{p}\right)$ is the one obtained through the reduction modulo $p$ map $\mathbf{G}_{\mathbb{Z}_{p}} \rightarrow \mathbf{G}_{\mathbb{F}_{p}}$, which is well-defined for almost all primes $p$.

The image of this map $\Phi$ is one $\mathbf{G}_{\mathbb{F}_{p}}$-orbit and the cardinality of the fibers of $\Phi$ does not depend on the fiber when it is non-empty. Let $v$ be a point in this image. The stabilizer of $v$ in the $\mathbb{F}_{p}$-group obtained from $\mathbf{G}$ by reduction modulo $p$, is a parabolic subgroup. Hence the orbit $\mathbf{M}$ of $v$ is a projective $\mathbb{F}_{p}$-variety. By Fact $(6.7)$, one has

$$
\mathbf{G}_{\mathbb{F}_{p}} \cdot v=\mathbf{M}_{\mathbb{F}_{p}} \text {. }
$$

The condition $\Lambda \subset \Lambda_{1}$ is equivalent to the condition $\Phi(\Lambda) \in \mathbf{M}_{\mathbb{F}_{p}}^{\prime}$ where $\mathbf{M}^{\prime}$ is the pro-

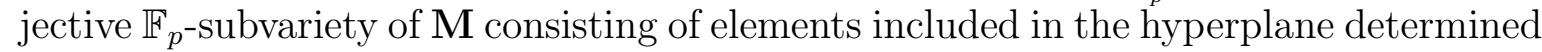
by $\Lambda_{1}$. Since the $\mathbb{Q}$-embedding of $\mathbf{G}$ is an absolutely irreducible $\mathbb{Q}$-representation, by Fact (6.6), for almost all $p$, the representation of $\mathbf{G}_{\mathbb{F}_{p}}$ in $\mathbb{F}_{p}^{N}$ is still an absolutely irreducible representation. Hence, $\mathbf{M}$ is not included in any hyperplane (in particular, the above parabolic is a proper parabolic subgroup) and one has

$$
\operatorname{dim}\left(\mathbf{M}^{\prime}\right)<\operatorname{dim}(\mathbf{M})
$$

We want to apply Fact 6.8 to these projective $\mathbb{F}_{p}$-varieties, for this we need to check its hypothesis. Note first that these projective varieties are included in the Grassmanian of $\mathbb{F}_{p}^{N}$ with $N$ fixed, hence using Plücker coordinates, in a projective space of dimension $N_{0}:=2^{N}$. The dimensions of $\mathbf{M}$ and $\mathbf{M}^{\prime}$ are also bounded by $N_{0}$. The degrees of $\mathbf{M}$ is bounded independently of $p$ and $a$ since these varieties are obtained by reduction modulo $p$ among finitely many flag subvarieties of the Grassmanian. Moreover $\mathbf{M}$ is 
absolutely irreducible being a homogeneous variety of $\mathbf{G}$. Since $\mathbf{M}^{\prime}$ is obtained from $\mathbf{M}$ by intersection with hyperplanes, the degree of $\mathbf{M}^{\prime}$ is bounded by the degree of $\mathbf{M}$. The number and the degrees of irreducible components of $\mathbf{M}^{\prime}$ are bounded independently of $a$, since the degree of $\mathbf{M}^{\prime}$ is the sum of the degrees of the irreducible components of $\mathbf{M}^{\prime}$. Therefore, by applying Fact 6.8 to $\mathbf{M}$ as well as to the irreducible components of $\mathbf{M}^{\prime}$, one has

$$
\frac{\#\left\{\Lambda \in \mathrm{T}_{a}\left(\Lambda_{0}\right) \mid \Lambda \subset \Lambda_{1}\right\}}{\operatorname{deg}(a)}=\frac{\# \mathbf{M}_{\mathbb{F}_{p}}^{\prime}}{\# \mathbf{M}_{\mathbb{F}_{p}}} \leq \frac{D_{0}}{p},
$$

for some constant $D_{0}$ independent of $a$.

Remark The same argument proves that, if $a$ is not in $\mathbf{G}_{\mathbb{Z}_{p}}$, then one has the inequality

$$
\operatorname{deg}(a) \geq p .
$$

In fact, this proof shows that $\operatorname{deg}(a)=\# \mathrm{~T}_{a}\left(\Lambda_{0}\right) \geq \# \mathbf{M}_{\mathbb{F}_{p}}$. But the number of $\mathbb{F}_{p^{-}}$ points of a non trivial flag variety of a semisimple $\mathbb{F}_{p}$-group is at least $p$.

Proof of Theorem 6.1 Later on, we will use this theorem only for $a$ in $\mathbf{G}_{\mathbb{Z}[1 / p]}$. In fact, the first part of the proof is to reduce it to the case where $a$ is in $\mathbf{G}_{\mathbb{Z}[1 / p]}$.

To explain this reduction, we recall that

- The quotient $\Gamma \backslash \mathbf{G}_{\mathbb{Q}}$ is the restricted product of the quotient spaces $U_{p} \backslash \mathbf{G}_{\mathbb{Q}_{p}}$, and $U_{p}=\mathbf{G}_{\mathbb{Z}_{p}}$ for almost all $p$.

- The Hecke orbit $\mathrm{T}_{a}\left(x_{0}\right)$ is a finite product $\prod_{p \in \operatorname{supp}(a)} \mathrm{T}_{a_{p}}\left(x_{0}\right)$ where $a=\prod_{p} a_{p}$ with $\operatorname{supp}\left(a_{p}\right)=\{p\}$ (Lemma 3.10.a) .

- If $x=\prod x_{p} \in \mathrm{T}_{a}\left(x_{0}\right)$ with $x_{p} \in \mathrm{T}_{a_{p}}\left(x_{0}\right)$ the multiplicities $m_{p}(x, \Delta)$ and $m_{p}\left(x_{p}, \Delta\right)$ are equal.

It follows that

$$
\frac{\# S_{a}\left(x_{0}, \Delta, p\right)}{\operatorname{deg}(a)}=\frac{\# S_{a_{p}}\left(x_{0}, \Delta, p\right)}{\operatorname{deg}\left(a_{p}\right)},
$$

which allows us to replace $a$ by $a_{p}$. From now on, we may assume that $a \in \mathbf{G}_{\mathbb{Z}[1 / p]}$.

By Lemma 3.2.b, we may replace $\Delta$ by the intersection of the Zariski closure of $\Delta$ with $\mathbf{G}_{\mathbb{Q}}$. Hence we assume $\Delta=\mathbf{H}_{\mathbb{Q}}$ where $\mathbf{H}$ is a $\mathbb{Q}$ subtorus of $\mathbf{G}$.

We can also suppose that $\mathbf{G}$ has trivial center. In fact, for any semisimple $\mathbb{Q}$-group $\mathbf{G}$, its center $\mathbf{Z}$ is finite. Moreover for almost all primes $p$, one has

$$
\mathrm{Z} \cap \mathbf{G}_{\mathbb{Q}_{p}} \subset \mathbf{G}_{\mathbb{Z}_{p}}
$$

and hence $\mathbf{G}_{\mathbb{Z}_{p}} \backslash \mathbf{G}_{\mathbb{Q}_{p}}$ injects in $(\mathbf{G} / \mathbf{Z})_{\mathbb{Z}_{p}} \backslash(\mathbf{G} / \mathbf{Z})_{\mathbb{Q}_{p}}$. This allows us to replace $\mathbf{G}$ by $\mathbf{G} / \mathbf{Z}$.

Note also that the statement of Proposition 6.1 does not depend on any $\mathbb{Q}$-embedding of $\mathbf{G}$. Hence using Fact (6.5), we may choose an absolutely irreducible $\mathbb{Q}$-embedding of $\mathbf{G}$ in $\mathbf{S L}_{\mathbf{N}}$. Now Theorem 6.1 with the constant $D=N D_{0}$ follows from Proposition 6.3 and Lemma 6.4 .

We note in the above proof that, if we exclude a finite set of primes $S=S(\Gamma, \Delta)$, the constant $D$ can be chosen independent of $\Gamma$ and $\Delta$, i.e. depending only on $\mathbf{G}$. 


\section{Proof of Theorem 1.1}

\subsection{Approximation of the weighted Hecke operator.}

Theorem 7.1. Let $\mathbf{G}, H$ be as in Theorem 1.1 and let $x_{0} H$ an $H$-orbit with finite volume with $x_{0}=\pi(e)$. Let $\Gamma \subset \mathbf{G}_{\mathbb{Q}}$ a congruence subgroup of the form $\Gamma=\mathbf{G}_{\mathbb{Q}} \cap \prod_{p} U_{p}$ for compact open subgroups $U_{p} \subset \mathbf{G}_{\mathbb{Q}_{p}}$. Let $a \in \mathbf{G}_{\mathbb{Q}}$ and $p \in \operatorname{supp}(a)$. Write $a=b c$ with $b$ and $c$ relatively prime and $\operatorname{supp}(c)=\{p\}$. For any $f \in \mathrm{C}_{c}(X)$, one has

$$
\left\|\widetilde{\mathrm{T}}_{a} f-\widetilde{\mathrm{T}}_{b}\left(\mathrm{~T}_{c} f\right)\right\|_{\infty} \leq \frac{D_{0}}{p}\|f\|_{\infty}
$$

where $\widetilde{\mathrm{T}}_{a}=\widetilde{\mathrm{T}}_{a}^{H}$ and $\widetilde{\mathrm{T}}_{b}=\widetilde{\mathrm{T}}_{b}^{H}$ are weighted Hecke operators with respect to $H$ and $D_{0}=D_{0}\left(\Gamma, H \cap \mathbf{G}_{\mathbb{Q}}\right)>0$ is a constant.

Indeed, $D_{0}=2 D$ where the constant $D$ is as in Theorem 6.1.

Proof Set $\Delta=H \cap \mathbf{G}_{\mathbb{Q}}$. Define $\delta f \in C(G)$ by

$$
\delta f:=\widetilde{\operatorname{deg}}(b) \operatorname{deg}(c) \widetilde{\mathrm{T}}_{b}\left(\mathrm{~T}_{c} f\right)-\widetilde{\operatorname{deg}}(a) \widetilde{\mathrm{T}}_{a}(f) .
$$

Since $b$ and $c$ are relatively primes, one has

$$
\delta f(e)=\sum_{x \in \mathrm{T}_{b}\left(x_{0}\right)} \sum_{y \in \mathrm{T}_{c}\left(x_{0}\right)}\left(\frac{1}{m(x)}-\frac{1}{m(x \cdot y)}\right) f(x \cdot y) .
$$

When $y$ is in $S_{c}\left(x_{0}, \Delta, p\right)$, the local multiplicity $\widehat{m}(y)=m_{p}(y)$ is equal to 1 , and by Lemma 3.11.c, one has $m(x . y)=m(x)$. When $y$ is not in $S_{c}\left(x_{0}, \Delta, p\right)$, it follows from Lemma 3.11.b that

$$
\left|\frac{1}{m(x)}-\frac{1}{m(x . y)}\right| \leq \frac{1}{m(x)} .
$$

Hence, using Theorem 6.1, one obtains

$$
\begin{aligned}
|\delta f(e)| & \leq \frac{D}{p} \operatorname{deg}(c) \sum_{x \in \mathrm{T}_{b}\left(x_{0}\right)} \frac{1}{m(x)}|f(x . y)| \\
& \leq \frac{D}{p} \widetilde{\operatorname{deg}(b)} \operatorname{deg}(c)\|f\|_{\infty} .
\end{aligned}
$$

By the $G$-equivariance, one gets the same upper bound at every point of $G$. Hence

$$
\|\delta f\|_{\infty} \leq \frac{D}{p} \widetilde{\operatorname{deg}}(b) \operatorname{deg}(c)\|f\|_{\infty} .
$$

Applying this inequality to the constant function, one has

$$
|\widetilde{\operatorname{deg}}(b) \operatorname{deg}(c)-\widetilde{\operatorname{deg}}(a)| \leq \frac{D}{p} \widetilde{\operatorname{deg}}(b) \operatorname{deg}(c) .
$$


Therefore,

$$
\begin{aligned}
& \left\|\mathrm{T}_{b}\left(\mathrm{~T}_{c} f\right)-\widetilde{\mathrm{T}}_{a} f\right\|_{\infty} \\
= & \left\|\frac{1}{\widetilde{\operatorname{deg}}(b) \operatorname{deg}(c)} \sum_{x \in \mathrm{T}_{b}\left(x_{0}\right)} \sum_{y \in \mathrm{T}_{c}\left(x_{0}\right)} \frac{f(x . y)}{m(x)}-\frac{1}{\widetilde{\operatorname{deg}(a)}} \sum_{x \in \mathrm{T}_{b}\left(x_{0}\right)} \sum_{y \in \mathrm{T}_{c}\left(x_{0}\right)} \frac{f(x . y)}{m(x . y)}\right\|_{\infty} \\
\leq & \frac{\|\delta f\|_{\infty}}{\widetilde{\operatorname{deg}}(b) \operatorname{deg}(c)}+\left|1-\frac{\widetilde{\operatorname{deg}}(a)}{\widetilde{\operatorname{deg}(b)} \operatorname{deg}(c)}\right|\left\|\widetilde{\mathrm{T}}_{a} f\right\|_{\infty} \\
\leq & \frac{2 D}{p}\|f\|_{\infty}
\end{aligned}
$$

which is the bound we were looking for.

7.2. Proof of Theorem 1.1. By Theorem 5.5, we only need to consider the case when $\# \operatorname{supp}(a)$ is not bounded. Letting $p_{a}:=\max (\operatorname{supp}(a))$, we may now assume that $p_{a} \rightarrow \infty$ as $\operatorname{deg}(a) \rightarrow \infty$. As in the proof of Theorem 5.5, we may assume that $x_{0}=\pi(e)$ and $\Gamma$ is of the form $G_{\mathbb{Q}} \cap \prod_{p} U_{p}$.

Let $f \in \mathrm{C}_{c}(X)$ with $\int_{X} f d \mu_{X}=0$. Applying Theorem 7.1 with $p_{a}$, one has, writing $a=b c$ with $b, c$ relatively prime and $\operatorname{supp}(c)=\left\{p_{a}\right\}$,

$$
\lim _{a \in S}\left\|\widetilde{\mathrm{T}}_{a} f-\widetilde{\mathrm{T}}_{b}\left(\mathrm{~T}_{c} f\right)\right\|_{\infty}=0 .
$$

We note that, since $X$ is compact, Theorem 2.1 implies that

$$
\lim _{\operatorname{deg}(c) \rightarrow \infty}\left\|\mathrm{T}_{c} f\right\|_{\infty}=0 .
$$

It is easy to see that $\operatorname{deg}(c) \geq p_{a}$ for all sufficiently large $p_{a}$. Hence as $\operatorname{deg}(a) \rightarrow \infty$, $\operatorname{deg}(c) \rightarrow \infty$.

Since $\widetilde{T}_{b}$ decreases the sup norm, one has

$$
\lim _{a \in S}\left\|\widetilde{\mathrm{T}}_{a} f\right\|_{\infty} \leq \lim _{a \in S}\left\|\mathrm{~T}_{c} f\right\|_{\infty}
$$

and hence

$$
\lim _{a \in S}\left\|\widetilde{T}_{a} f\right\|_{\infty}=0 \text {. }
$$

This finishes the proof of Theorem 1.1 by Lemma 2.6.

Remark The assertion (7.2) is the only place in the proof of Theorem 1.1 where we use the cocompactness hypothesis on $\Gamma$. Note that in the case when $\# \operatorname{supp}(a)$ is unbounded, our proof of Theorem 1.1 works for the case $H$ compact as well.

Since $\lambda_{a}(X)=\widetilde{\operatorname{deg}}^{H}(a) \cdot \lambda_{H}\left(x_{0} H\right)$, the following corollary shows that $\operatorname{deg}(a) \rightarrow \infty$ if and only if $\lambda_{a}(X) \rightarrow \infty$ as mentioned in the introduction. 
Corollary 7.3. Let $\mathbf{G}, H$ be as in Theorem 1.1 and $\Gamma$ be any congruence subgroup. For a sequence $\left\{a \in G_{\mathbb{Q}}\right\}$, one has the equivalence:

$$
\operatorname{deg}(a) \rightarrow \infty \Longleftrightarrow \widetilde{\operatorname{deg}}^{H}(a) \rightarrow \infty
$$

Proof Since $\widetilde{\operatorname{deg}}^{H}(a) \leq \operatorname{deg}(a)$ clearly, we only have to prove the implication $\Longrightarrow$.

When $\# \operatorname{supp}(a)$ is bounded, we have seen in Proposition 4.5 that the multiplicities are bounded by some integer $m_{0}$. Hence $\operatorname{deg}(a) \leq m_{0} \widetilde{\operatorname{deg}}(a)$.

Suppose \# $\operatorname{supp}(a)$ goes to infinity. By Lemma 3.7, one has

$$
\widetilde{\operatorname{deg}}(a) \geq \prod_{p} \widehat{\operatorname{deg}}_{p}(a)
$$

where $\widehat{\operatorname{deg}}_{p}(a)=\sum_{x \in T_{a_{p}}\left(x_{0}\right)} \frac{1}{m_{p}\left(x, H \cap \mathbf{G}_{\mathbb{Q}}\right)}$. Letting $p_{a}=\max (\operatorname{supp}(a))$, one obtains from 6.1 that for $p_{a}>D_{0}$

$$
\widetilde{\operatorname{deg}}(a) \geq \widehat{\operatorname{deg}}_{p_{a}}(a) \geq\left(1-\frac{D}{p_{a}}\right) \operatorname{deg}\left(a_{p_{a}}\right) \geq \frac{1}{2} \operatorname{deg}\left(a_{p_{a}}\right) \geq p_{a} / 2 .
$$

To prove the last inequality, just use the remark following the proof of Lemma 6.4.

In both cases, $\widetilde{\operatorname{deg}}(a)$ goes to infinity.

\section{Duality AND Double Classes}

8.1. Hecke orbits on $G / H$. Corollary 1.2 will be a consequence of Theorem 1.1 and of the following proposition. Recall that a Radon measure on a locally compact Hausdorff space simply means a locally finite regular Borel measure.

Proposition 8.1. Let $G$ be a locally compact group. Let $\Gamma$ and $H$ be two closed subgroups of $G$. Fix a right Haar measure $\lambda_{\Gamma}$ on $\Gamma$ and a left Haar measure $\lambda_{H}$ on $H$. Then there are homeomorphisms among the following spaces equipped with the weak * topologies

$$
\left\{\begin{array}{c}
\text { right } H \text {-invariant } \\
\text { Radon measures } \\
\text { on } \Gamma \backslash G \\
\mu
\end{array}\right\} \leftrightarrow\left\{\begin{array}{c}
\text { left } \Gamma \text {-invariant and } \\
\text { right } H \text { - invariant } \\
\text { Radon measures on } G
\end{array}\right\} \leftrightarrow \leftrightarrow \leftrightarrow\left\{\begin{array}{c}
\text { left } \Gamma \text {-invariant } \\
\text { Radon measures } \\
\text { on } G / H \\
\nu
\end{array}\right\}
$$

given by, for any $f \in \mathrm{C}_{c}(G)$,

$$
\int_{\Gamma \backslash G}\left(\int_{\Gamma} f(\gamma g) d \lambda_{\Gamma}(\gamma)\right) d \mu(\Gamma g)=\int_{G} f(g) d \nu(g)=\int_{G / H}\left(\int_{H} f(g h) d \lambda_{H}(h)\right) d \rho(g H)
$$

This proposition follows immediately by applying the following general lemma twice. 
Lemma 8.2. Let $G$ be a locally compact group and $H$ a closed subgroup of $G$. Fix a left Haar measure $\lambda_{H}$ on $H$. Then there is a homeomorphism between the following spaces

$$
\left\{\begin{array}{c}
\text { right } H \text {-invariant } \\
\text { Radon measures on } G
\end{array}\right\} \leftrightarrow\left\{\begin{array}{c}
\text { Radon measures } \\
\text { on } G / H \\
\rho
\end{array}\right\}
$$

given by, for any $f \in \mathrm{C}_{c}(G)$,

$$
\int_{G} f(g) d \nu(g)=\int_{G / H}\left(\int_{H} f(g h) d \lambda_{H}(h)\right) d \rho(g H) .
$$

Proof The map $I: C_{c}(G) \rightarrow C_{c}(G / H)$ defined by

$$
I(f)(g H)=\int_{H} f(g h) d \lambda_{H}(h)
$$

is surjective [Ra, Lemma 1.1]. For a given right $H$-invariant Radon measure $\nu$ on $G$, define

$$
\rho(I(f)):=\int_{G} f(g) d \nu(g)=\nu(f)
$$

for $f \in C_{c}(G)$. To check this is well defined, suppose that $I(f)=0$. Then

$$
\int_{G} I(f)(g H) d \nu(g)=0 .
$$

By applying Fubini's lemma, one has $\int_{H} \int_{G} f(g h) d \nu(g) d \lambda_{H}(h)=0$. Since $\nu$ is right $H$-invariant, it follows that $\int_{H} \nu(f) d \lambda_{H}(h)=0$ and hence $\nu(f)=0$. Hence $\rho$ defines a Radon measure on $G / H$. Now for a given $\rho$, one can define a right $H$-invariant measure $\nu$ on $G$ as above. Clearly this map $\nu \rightarrow \rho$ is a homeomorphism.

Note that if $\Gamma$ is a closed subgroup of $G$, then the left $\Gamma$-invariant measures $\nu$ correspond to the left $\Gamma$-invariant measures $\rho$ in the above lemma, and hence Proposition 8.1 follows.

Proof of Corollary 1.2 Let $d g$ and $d h$ denote the Haar measures on $G$ and $H$ respectively so that $\Gamma \backslash G$ and $(\Gamma \cap H) \backslash H$ have both volume 1 . Let $\lambda$ be the $G$-invariant measure $\lambda$ on $G / H$ to be the "quotient" of $d g$ by $d h$, i.e., for any $f \in C_{c}(G)$, we have

$$
\int_{G} f d g=\int_{G / H} \int_{H} f(g h) d h d \lambda[g] .
$$

In this case, one has $\lambda_{a}(X)=\widetilde{\operatorname{deg}}(a)$ and one can take

$$
c_{a}=\widetilde{\operatorname{deg}}(a) .
$$

In fact, according to the homeomorphism given in Proposition 8.1, each $H$-invariant measure $\mu_{a}=c_{a}^{-1} \lambda_{a}$ on $X=\Gamma \backslash G$ correspond to the $\Gamma$-invariant measure $\rho_{a}$ on $V=$ 
$G / H$ given by

$$
\rho_{a}(\Omega)=\frac{1}{c_{a}} \#\left(V_{a} \cap \Omega\right)
$$

for any measurable subset $\Omega \subset V$, and the measure $\mu_{X}$ on $X=\Gamma \backslash G$ correspond to the measure $\lambda$ on $V=G / H$. By Theorem 1.1, the sequence $\mu_{a}$ converges to $\mu_{X}$, and hence the sequence $\rho_{a}$ converges to $\lambda$.

Remark 8.3. Corollary 1.2 is still true even if $\mathbf{G}$ is not necessarily simply connected and $\Gamma$ is not necessarily cocompact if one take $G=\mathbf{G}(\mathbb{R})^{0}$ and $a \in G_{\mathbb{Q}}$ with \# $\operatorname{supp}(a)$ uniformly bounded. The same proof works, replacing Theorem 1.1 by Theorem 5.5.

Proof of Corollary 1.3 Denote by $H$ the centralizer of $s$ in $G$. Then $H$ is a noncompact torus, which is $\mathbb{Q}$-anisotropic. The last condition is equivalent to saying the $H$-orbit $[e] H$ is compact in $\Gamma \backslash G$. The homogeneous space $V=G / H$ can be identified with $\mathcal{C}(s)$ via the map $g \mapsto g s g^{-1}$, and this identification carries $V_{a}$ to the set

$$
\left\{t \in \mathcal{C}(s) \mid t=g s g^{-1} \text { for some } g \in \Gamma a \Gamma\right\} .
$$

Hence this corollary is a special case of Corollary 1.2.

8.2. Conjugacy classes of rational matrices. We can now come back to our concrete example.

Proof of Proposition 1.4 Denote by $M_{0}$ the companion matrix associated to the polynomial $P(T)=T^{N}+a_{N-1} T^{N-1}+\cdots+a_{0}$ with $N \geq 3$ :

$$
M_{0}:=\left(\begin{array}{cccc}
0 & 1 & \cdots & 0 \\
0 & 0 & \cdots & 0 \\
\vdots & & & 1 \\
-a_{0} & -a_{1} & \cdots & -a_{N-1}
\end{array}\right) .
$$

Let $\mathrm{GL}_{N}^{+}(\mathbb{R})$ denote the subgroup of $\mathrm{GL}_{N}(\mathbb{R})$ consisting of all matrices of positive determinant. As is well known, $V$ (resp. $V_{\mathbb{Q}}$ ) is the conjugacy class of $M_{0}$ in $\mathrm{GL}_{N}^{+}(\mathbb{R})$ (resp. $\left.\mathrm{GL}_{N}^{+}(\mathbb{Q})\right)$. Note that the centralizer of $M_{0}$ in $\mathbf{P G L}$ is a maximal torus which is $\mathbb{R}$-isotropic since $N \geq 3$ and $\mathbb{Q}$-anisotropic since $P$ is irreducible (cf. [EMS]). Hence if we set $G=\mathrm{PGL}_{N}^{+}(\mathbb{R})$ and $H$ to be the centralizer of $M_{0}$ in $G$, then $G$ and $H$ satisfies the assumptions in Theorem 5.5 (with $\mathbf{G}=\mathbf{P G L}_{\mathbf{N}}$ ) and we may identify $V$ with $G / H$ via the map $g M_{0} g^{-1} \mapsto g H$. Now by Remark 8.3, we may apply Corollary 1.2 to this case with $\Gamma=\mathrm{PGL}_{N}^{+}(\mathbb{Z})=\mathrm{SL}_{N}(\mathbb{Z})$, provided \# $\operatorname{supp}(a)$ are uniformly bounded.

Set $a_{m}=\operatorname{diag}\left(m_{1}, \ldots, m_{N}\right)$. The condition on $m$ in Proposition 1.4 is equivalent to saying that \# $\operatorname{supp}\left(a_{m}\right)$ are uniformly bounded for all $m$. Therefore we only need to check that

$$
V_{m}=\operatorname{Ad}\left(\mathrm{SL}_{N}(\mathbb{Z}) a_{m} \mathrm{SL}_{N}(\mathbb{Z})\right)\left(M_{0}\right)
$$


Set

$$
\mathcal{L}:=\{\Lambda \mid \text { finite index } \mathbb{Z} \text {-submodule of } \mathbb{Z}[\alpha]\} / \sim
$$

where $\Lambda_{1} \sim \Lambda_{2}$ if and only if $\Lambda_{1}=u \Lambda_{2}$ for some non-zero $u \in \mathbb{Q}(\alpha)$.

In the space $\mathcal{L}$, we may define, for each $m=\left(m_{1}, \cdots, m_{N}\right)$, the Hecke orbit $\mathrm{T}_{m}(\mathbb{Z}[\alpha])$ of $\mathbb{Z}[\alpha]$ to be

$$
\mathrm{T}_{m}(\mathbb{Z}[\alpha]):=\left\{[\Lambda] \in \mathcal{L} \mid \mathbb{Z}[\alpha] / \Lambda \simeq \oplus_{i=1}^{N} \mathbb{Z} / m_{i} \mathbb{Z}\right\} .
$$

Define the map $\phi: V_{\mathbb{Q}} \rightarrow \mathcal{L}$ by $M \mapsto\left[\Lambda_{\xi_{M}}\right]$ where $\xi_{M}=\left(\xi_{1}, \ldots, \xi_{N}\right) \in \mathbb{Z}[\alpha]^{N}$ is an eigenvector of $M$ for $\alpha$. Since the eigenvector $\xi_{M}$ of $M$ for $\alpha$ is unique up to multiplication by $\mathbb{Q}(\alpha), \phi$ is well defined. Observing that

$$
V_{m}=\phi^{-1}\left(\mathrm{~T}_{m}(\mathbb{Z}[\alpha])\right),
$$

the following lemma provides (8.4).

Lemma 8.5. The map $\phi: V_{\mathbb{Q}} \rightarrow \mathcal{L}$ is a surjection such that for any $m=\left(m_{1}, \cdots, m_{N}\right)$ with $m_{i} \in \mathbb{N}, m_{i+1} \mid m_{i}$ for all $i$ and $m_{N}=1$,

$$
\operatorname{Ad}\left(\mathrm{SL}_{N}(\mathbb{Z}) a_{m} \mathrm{SL}_{N}(\mathbb{Z})\right)\left(M_{0}\right)=\phi^{-1}\left(\mathrm{~T}_{m}(\mathbb{Z}[\alpha])\right)
$$

Proof If we set $\xi_{0}:=\left(1, \alpha, \ldots, \alpha^{N-1}\right)^{t} \in \mathbb{Z}[\alpha]^{N}$, then $M_{0} \xi_{0}=\alpha \xi_{0}$. Let $[\Lambda] \in \mathcal{L}$. Since $\left(1, \alpha, \ldots, \alpha^{N-1}\right)$ is a basis of the $\mathbb{Z}$-module $\mathbb{Z}[\alpha]$, there exists $g=\left(g_{i j}\right) \in \mathrm{GL}_{N}^{+}(\mathbb{Q}) \cap$ $\mathcal{M}_{N}(\mathbb{Z})$ such that $\left(\xi_{1}, \cdots, \xi_{N}\right)$ is a basis for $\Lambda$ where $\left(\xi_{1}, \cdots, \xi_{N}\right)^{t}=g \xi_{0}$. Clearly, $g \xi_{0}$ is an eigenvector for $M:=g M_{0} g^{-1}$ with respect to the eigenvalue $\alpha$. Hence $\phi\left(g M_{0} g^{-1}\right)=[\Lambda]$. This shows $\phi$ is surjective.

By the elementary divisor theory, any $g \in \mathrm{GL}_{N}^{+}(\mathbb{Q})$ belongs to $\mathrm{SL}_{N}(\mathbb{Z}) a_{m} \mathrm{SL}_{N}(\mathbb{Z})$ where $a_{m}=\operatorname{diag}\left(m_{1}, \cdots, m_{N}\right)$ is the unique diagonal element with the property that $m_{i} \in \mathbb{N}, m_{i+1} \mid m_{i}$ for all $i$ and $m_{N}=1$. Moreover, by the structure theorem for $\mathbb{Z}$ submodules of $\mathbb{Z}^{N}$, for $\xi=g \xi_{0}$ with $g \in \mathrm{GL}_{N}^{+}(\mathbb{Q})$, the quotient $\mathbb{Z}[\alpha] / \Lambda$ is isomorphic to $\oplus_{i=1}^{N} \mathbb{Z} / m_{i} \mathbb{Z}$ for some $\Lambda \in\left[\Lambda_{\xi}\right]$ if and only if $g \in \operatorname{SL}_{N}(\mathbb{Z}) a_{m} \operatorname{SL}_{N}(\mathbb{Z})$. This finishes the proof.

It is easy to see that the same proof holds for $N=2$ and $\alpha \in \mathbb{R}$ as well. However the arguments in this paper does not prove the case of $N=2$ with $\alpha \in \mathbb{C}$, which we believe to be still true.

\section{REFERENCES}

[Bo] A. Borel Linear algebraic groups, GTM 126, Springer (1991).

[BH] A. Borel and Harish-Chandra Arithmetic subgroups of algebraic groups, Ann. Math., Vol 75, 1962, pp. $485-535$.

[COU] L. Clozel, H. Oh and E. Ullmo Hecke operators and equidistribution of Hecke points, Inv. Math. 144 (2003) p.327-351.

[EMS] A. Eskin, S. Mozes, N. Shah Unipotent flows and counting lattice points on homogeneous varieties, Annals of Math. 143 (1996) p.149-159.

[EO1] A. Eskin, H. Oh Ergodic theoretic proof of equidistribution of Hecke points, To appear in Erg. The. \& Dyn. Sys.

[EO2] A. Eskin, H. Oh Representations of integers by an invariant polynomial and unipotent flows, Preprint (2003). 
[GO] W. T. Gan, H. Oh Equidistribution of Integer points on a family of homogeneous varieties: A problem of Linnik, Compos. Math. 136 (2003) p.325-352.

[LW] S. Lang, A. Weil Number of points of varieties in finite fields, Amer. J. Math. 76 (1954) p.819827.

[LM] C. Latimer, C. MacDuffee A correspondence between classes of ideas and classes of matrices, Ann. of Math. 34 (1933), 313-316

[Li] Y. Linnik Additive problems and eigenvalues of the modular operators, Proc. Int. Cong. Math. (1962) p.270-284.

[PR] V. Platonov, A. Rapinchuk Algebraic groups and number theory, Acad. Press (1994).

[PR1] G. Prasad, M. S. Raghunathan Cartan subgroups and lattices in semisimple groups, Annals of Math., 96 (1972), 296-317

[Ra] M. Raghunathan Discrete subgroups of Lie groups, Springer (1972).

[Sa] P. Sarnak Diophantine problems and linear groups, Proc. Int. Cong. Math. 1 (1990) p.459-471.

[T] O. Taussky On a theorem of a Latimer and MacDuffee, Canad. J. Math (1949), p. 300-302

[Ti] J. Tits Représentations linéaires irréductibles d'un groupe réductif sur un corps quelconque, J. Reine Angew. Math. 247 (1971) p.196-220.

[S] N. Shah Counting integral matrices with a given characteristic polynomial Ergodic theory and Harmonic analysis, Mumbai, 62 (2000), p. 386-412

[Zi] R. Zimmer Ergodic theory and semisimple groups, Birkhäuser, (1984)

DMA-ENS 45 RUE D'Ulm PARIS 75005

E-mail address: benoist@dma.ens.fr

Math 253-37, Caltech, Pasadena, CA 91125

E-mail address: heeoh@its.caltech.edu 